\title{
Kizilcahamam Flora of Soguksu National Park
}

\author{
Omer Eyuboglu \\ Faculty Member of Ahievran University, \\ Faculty of Education, Department of Science Education/Turkey \\ E-mail: oeyuboglu@ahievran.edu.tr
}

\begin{abstract}
This research covers the flora of Soguksu National Park, Kizilcahamam. 7 families, 276 genera, 4 subspecies, and 3 varieties were determined with the evaluation of 1064 plant samples collected in the study area during 1989 and 1990. The total number of taxa was 481 and 49 of them were endemic in Turkey. The distribution and rates of the species by phytogeographical regions are as follows: 71 (14.8) European - Siberian components, $11(2.3 \%)$ euxinic components, 76 (15.8\%) Iranian - Turanian components, $31(6.5 \%)$ Mediterranean components, and 292 (60.9\%) unknown components or cosmopolits. 37 of the total 481 taxa were new for A4 grid square.
\end{abstract}

Key Words: Kizilcahamam, Soguksu National Park

DOI: $10.7176 / J S T R / 5-2-43$

\section{INTRODUCTION}

Despite the studies conducted on Turkey's flora started by the beginning of the last century, it accelarated with the publication of the work of Davis titled "Flora of Turkey and Eastern Aegean Islands" in 1965. The number of researches conducted by Turkish botanists climbed especially after 1970s due to the contributions of the published volumes of Davis's flora and the elevated number of universities throughout Turkey and increased number of researchers conducting floristic studies in these universities. The entirety of the study area of Soğuksu National Park Forest, Kızılcahamam is situated within the limits of Ankara City, covering an area of 1050 hectares. The field is a volcanic field filled with regional forests and thermal springs and cold water reservoirs in its vicinity.

The National Park limits cross the shoulders and a portion of it is surrounded by barbed wire against the grazing risk. It is $7 \mathrm{~km}$ away from the nearest village and the elevations starting at $1030 \mathrm{~m}$ in the National Park field reach $1776 \mathrm{~m}$, the heighest point, at Tolubelen Hill.

Based on the grid system used in Davis's work "Flora of Turkey" and the samples collected by us, there were 37 plant species that were new for A4 grid square (within A4 square grid of Soğuksu National Park), belonging to the families published in 10 volumes of the flora.

By determining the flora of the National Park in this study, we think that we have contributed to the flora of Ankara City and Turkey.

\section{BACKGROUND}

\section{Geographical Aspect of the Study Area}

Soğuksu National Park is situated in the Middle Anatolian Region of Turkey to the west of Kizılcahamam County. The National Park is $1 \mathrm{~km}$ away from the County center and $80 \mathrm{~km}$ away from Ankara, extending between $40^{\circ} 31^{\prime} 26^{\prime \prime}-40^{\circ} 34^{\prime} 13^{\prime \prime}$ northern latitudes and $32^{\circ} 35^{\prime} 10^{\prime \prime}-32^{\circ} 39^{\prime} 31^{\prime \prime}$ eastern longtitudes. Though the part of the National Park, where activities are held, starts to the west of the County, the main border crosses Kocaçay Creek, running in parallel to Ankara-Istanbul highway that passes through the eastern part of the County, and making the eastern border of the area. The horizon line, starting at Çekeş junction and connecting Yanik Shoulder and Tolubelen Hill, forms the northern border of the area. Tolubelen Hill and Göllü and Incegeliş Shoulders to the west and Harmandoruk Hill and Uzunkavaklık Shoulder to the south make up the borders of the National Park (Figure 1). 


\section{Topographical Structure and Geology}

Despite there are no major mountains in the National Park, there are some high hills in the valleys, as separeted from each other. The average altitude of $1100 \mathrm{~m}$ by Kizilcahamam County reaches $1776 \mathrm{~m}$ at Tolubelen Hill positioned to the west. The other critical altitudes are Harmandoruk Hill of $1645 \mathrm{~m}$, Keltepe Hill of 1350 m, Incegeliş Shoulder of 1600 m, Göllü Shoulder of 1630 m, and Çakmağın Doruk Hill of 1530 m (Figure 1).

Kocaçay Creek, making up the eastern border of the area, runs continuously in summers and winters. Although Soğuksu Creek, cutting through the middle of the National Park, is another significant creek in the National Park, it dries in summers. Another valley to the west of the area is Cehennemdere but, this Creek is situated to the west of the National Park border.

There is a depression covered with water up until the mids of summer at Göllü locality of the National Park, filled with hygrophile vegetation. Snow waters collected in this depression dries after the month of July.

The area's geological structure is rather homogenous, made of andesite and dacite extrusive rocks, and some parts include a neogenic field with a volcanic sahara intermediate layer.

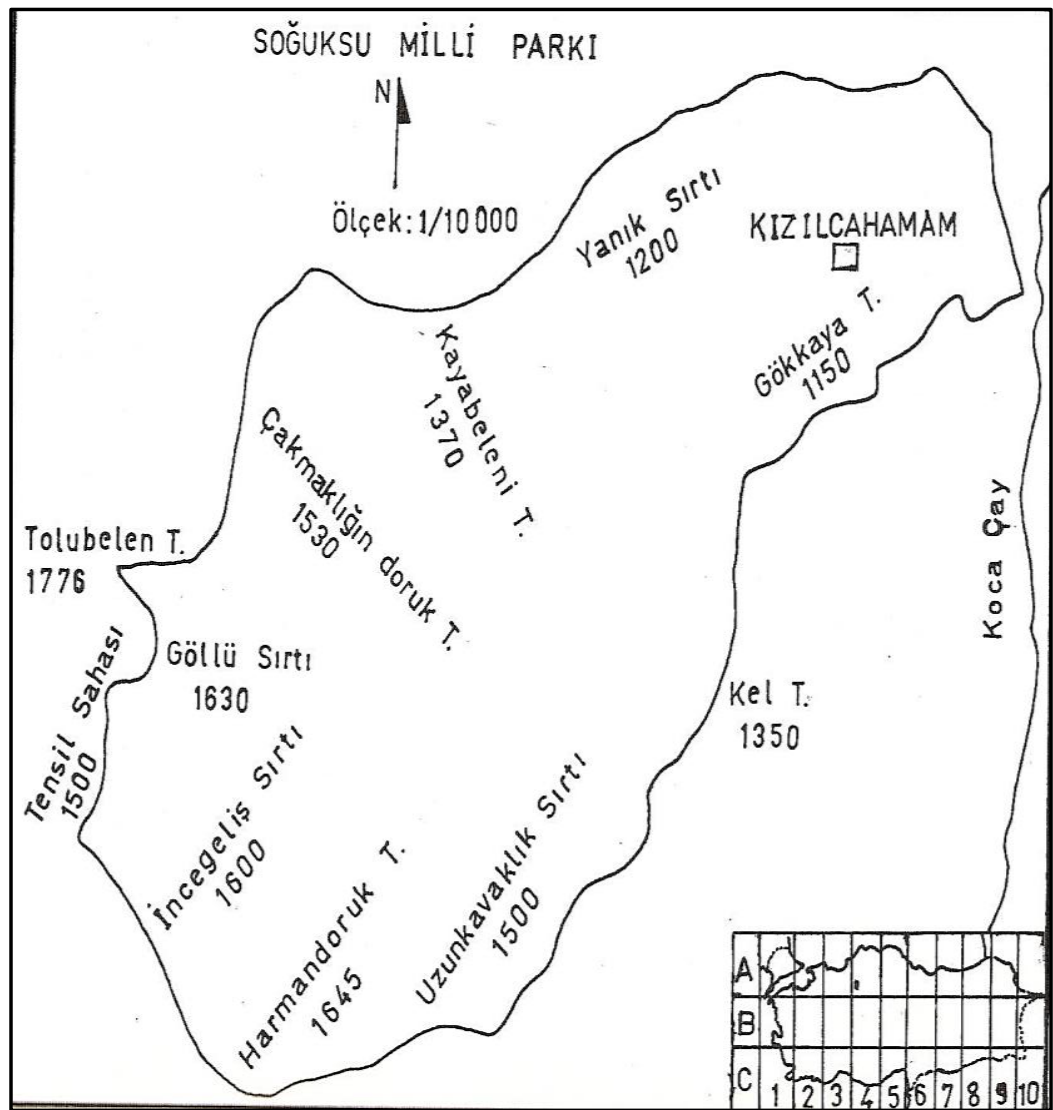

Soğuksu National Park, scale: 1/10000, Tensil field, Uzzunkavaklık Shoulder, Yanık Shoulder, Kocaçay Creek

Figure1. Geographical Map of the Area

\section{CLIMATE}

Soğuksu National Park is situated in semi-arid, very cold Mediterranean bioclimatic stage (Table 1).

\section{Temperature}

Annual average temperature is $10.2^{\circ} \mathrm{C}$. The hottest month in the study area is August $\left(30{ }^{\circ} \mathrm{C}\right)$ and the coldest month is January $\left(-5^{\circ} \mathrm{C}\right)$ (Tables $\left.2,3,4\right)$. 


\section{Precipitation}

The annual average precipitation is $564.4 \mathrm{~mm}$, with maximum precipitation of $85.3 \mathrm{~mm}$ received in December and minimum precipitation of $14.9 \mathrm{~mm}$ received in August (Table 5). Maximum precipitation is received in winter $(235.2 \mathrm{~mm})$ and minimum precipitation is received in summer $(73.7 \mathrm{~mm})$ (Table 6). Average relative humidity is $65 \%$, with maximum annual average of $77 \%$ in December and minimum annual average of 51\% in August (Table 7).

The number of annual average foggy days is 4.6, with April, June, July and August having no foggy days, and December has the maximum number of foggy days. The number of snowy days is 12.6 according to the 41-year annual averages and the average number of days covered with snow is 14.6, the number of frost days is 88.6. The climatic diagram of Kizılcahamam is shown in Figure 2.

\section{Prevailing Winds}

Direction of the prevailing wind was North-eastern with the blowing number of 5102 and northern with the blowing number of 3757. In terms of the blowing force, the major directions are the southernsoutheastern $(3.8 \mathrm{~m} / \mathrm{sec})$ and north-northeastern $(3.7 \mathrm{~m} / \mathrm{sec})$ (Table 8$)$.

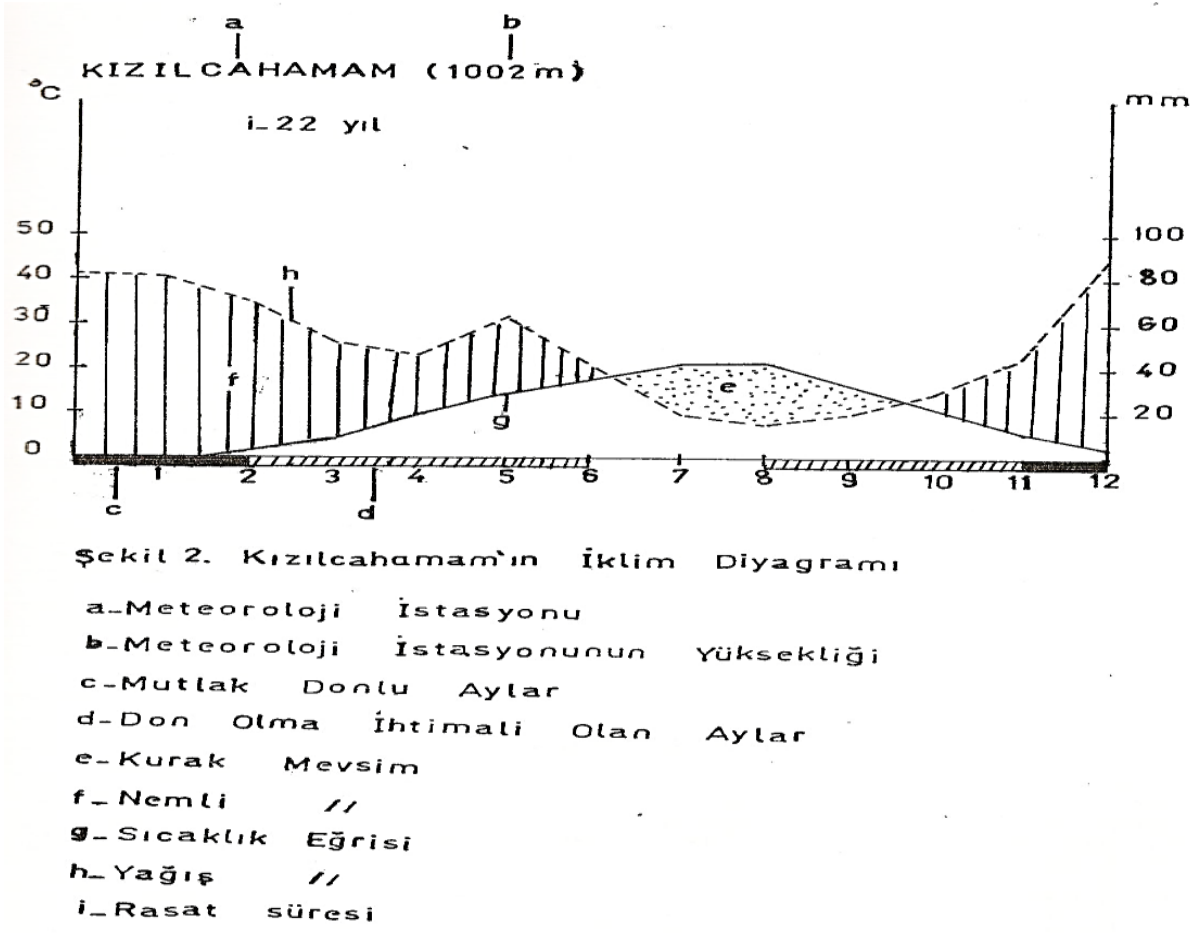

a-Meteorology station / b-Height of meteorology station / c- Months with absolute frost / d - Months with possible frost occurrence / e - Dry season / f- Humid season / g - Temperature curve / hPrecipitation curve / i- Observation period

Table 1. Kizılcahamam's Climatic Type

\begin{tabular}{|c|c|c|c|c|c|c|}
\hline STATION & $\mathrm{Q}$ & $\mathrm{m}$ & $\mathrm{M}$ & $\mathrm{PE}$ & $\mathrm{S}$ & CLIMATE \\
\hline Kizilcahamam & 56,4 & $-5^{\circ} \mathrm{C}$ & $30^{\circ} \mathrm{C}$ & 73,7 & 2,5 & $\begin{array}{c}\text { Semi-arid, } \\
\text { very cold } \\
\text { Meditarrenean } \\
\text { climate }\end{array}$ \\
\hline
\end{tabular}

P: Annual precipitation amount $(\mathrm{mm})$

$\mathrm{S}$ : Aridness index $\left(\mathrm{mm} /{ }^{\circ} \mathrm{C}\right)$

PE: Total three-month summer precipitation amount $(\mathrm{mm})$

$\mathrm{M}$ : Average maximum temperature of the hottest month $\left({ }^{\circ} \mathrm{C}\right)$

$\mathrm{M}$ : Average minimum temperature of the colest month of the year $\left({ }^{\circ} \mathrm{C}\right)$ 
International Journal of Scientific and Technological Research

ISSN 2422-8702 (Online), DOI: 10.7176/JSTR/5-2-43

Vol.5, No.2, 2019

Table 2. Monthly and annual average temperature

\begin{tabular}{|c|c|c|c|c|c|c|c|c|c|c|c|c|c|c|}
\hline \multirow{2}{*}{ STATION } & \multirow{2}{*}{$\begin{array}{l}\text { HEIGHT } \\
\text { (m) }\end{array}$} & \multicolumn{12}{|c|}{ MONTHS } & \multirow{2}{*}{$\begin{array}{c}\text { Annual } \\
\text { average }\left({ }^{\circ} \mathrm{C}\right)\end{array}$} \\
\hline & & I & II & III & IV & V & VI & VII & VIII & IX & X & XI & XII & \\
\hline Kizılcahamam & 1002 & $-0,2$ & $-0,3$ & 4,0 & 9,9 & 14,2 & 17,7 & 21,1 & 21,0 & 16,0 & 10,1 & 6,1 & 2,5 & 10,5 \\
\hline
\end{tabular}

Table 3. Monthly and annual maximum temperature averages

\begin{tabular}{|c|c|c|c|c|c|c|c|c|c|c|c|c|c|c|}
\hline \multirow{2}{*}{ STATION } & \multirow{2}{*}{$\begin{array}{l}\text { HEIGHT } \\
\text { (m) }\end{array}$} & \multicolumn{12}{|c|}{ MONTHS } & \multirow{2}{*}{$\begin{array}{c}\text { Annual } \\
\text { Average }\left({ }^{\circ} \mathrm{C}\right)\end{array}$} \\
\hline & & I & II & III & IV & $\mathrm{V}$ & VI & VII & VIII & IX & $X$ & XI & XII & \\
\hline Kızılcahamam & 1002 & 4,9 & 5,3 & 10,8 & 17,4 & 21,9 & 26,6 & 29,3 & 30,0 & 25,4 & 19,2 & 13,9 & 7,1 & 17,6 \\
\hline
\end{tabular}

Table 4. Annual minimum temperature averages

\begin{tabular}{|c|c|c|c|c|c|c|c|c|c|c|c|c|c|c|}
\hline \multirow{2}{*}{ STATION } & \multirow{2}{*}{$\begin{array}{l}\text { HEIGHT } \\
\text { (m) }\end{array}$} & \multicolumn{12}{|c|}{ MONTHS } & \multirow{2}{*}{$\begin{array}{l}\text { Annual Average } \\
\left({ }^{\circ} \mathrm{C}\right)\end{array}$} \\
\hline & & I & II & III & IV & V & VI & VII & VIII & IX & $X$ & XI & XII & \\
\hline Kızılcahamam & 1002 & $-4,1$ & $-5,0$ & $-1,6$ & 3,0 & 6,8 & 9,3 & 12,3 & 11,6 & 7,5 & 3,2 & 0,4 & $-0,9$ & 3,5 \\
\hline
\end{tabular}

Table 5. Monthly and annual average precipitation

\begin{tabular}{|c|c|c|c|c|c|c|c|c|c|c|c|c|c|c|}
\hline & \multicolumn{9}{|c|}{ MONTHS } & \multicolumn{1}{c|}{ Annual } \\
STATION & $\begin{array}{c}\text { HEIGHT } \\
\text { (m) }\end{array}$ & I & II & III & IV & V & VI & VII & VIII & IX & X & XI & XII & Average \\
\hline Kizilcahamam & 1002 & 80,5 & 69,4 & 51,1 & 45,8 & 62,6 & 41,0 & 17,8 & 14,9 & 18,5 & 27,2 & 44,3 & 85,3 & 564,4 \\
\hline
\end{tabular}


Table 6. Precipitation values by seasons

\begin{tabular}{|c|c|c|c|c|c|c|c|c|c|c|}
\hline \multirow{3}{*}{$\begin{array}{c}\text { STATION } \\
\text { Kizilcahamam }\end{array}$} & \multirow{3}{*}{\begin{tabular}{|c}
$\begin{array}{c}\text { HEIGHT } \\
\text { (M) }\end{array}$ \\
1002
\end{tabular}} & \multicolumn{8}{|c|}{ Precipitation Values by Seasons } & \multirow{2}{*}{$\begin{array}{c}\text { Annual } \\
\text { average } \\
\text { precipitation }\end{array}$} \\
\hline & & \multicolumn{2}{|c|}{ Winter \% } & \multicolumn{2}{|c|}{ Spring $\%$} & \multicolumn{2}{|c|}{ Summer \% } & \multicolumn{2}{|c|}{ Fall \% } & \\
\hline & & 235,2 & 41,6 & 165,5 & 29,2 & 73,7 & 12,9 & 90,0 & 15,9 & 564,4 K.İ.S.Y. \\
\hline
\end{tabular}

Table 7. Partial humidity average values

\begin{tabular}{|c|c|c|c|c|c|c|c|c|c|c|c|c|c|c|}
\hline \multirow{2}{*}{ STATION } & \multirow{2}{*}{$\begin{array}{l}\text { HEIGHT } \\
\text { (m) }\end{array}$} & \multicolumn{12}{|c|}{ MONTHS } & \multirow{2}{*}{$\begin{array}{c}\text { Annual } \\
\text { Average }(\%)\end{array}$} \\
\hline & & I & II & III & IV & $\mathrm{V}$ & VI & VII & VIII & IX & $\mathrm{X}$ & XI & XII & \\
\hline Kizılcahamam & 1002 & 76 & 76 & 68 & 64 & 64 & 61 & 54 & 51 & 57 & 65 & 72 & 77 & 65 \\
\hline
\end{tabular}

Table 8. Wind Direction

\begin{tabular}{|c|c|c|c|c|c|c|c|c|c|c|c|c|c|c|}
\hline \multirow{2}{*}{ STATION } & \multirow{2}{*}{$\begin{array}{l}\text { HEIGHT } \\
\text { (m) }\end{array}$} & \multicolumn{12}{|c|}{ MONTHS } & \multirow{2}{*}{$\begin{array}{l}\text { Annual } \\
\text { Average }\end{array}$} \\
\hline & & I & II & III & IV & V & VI & VII & VIII & IX & $X$ & XI & XII & \\
\hline Kızılcahamam & 1002 & SE & SE & SE & SE & $\mathrm{NW}$ & NW & $\mathrm{NE}$ & NW & NW & SE & SE & SE & SE \\
\hline
\end{tabular}

SE : South east

NW : North west

NE : North east

\section{VEGETATION}

The study area was a passage zone extending between European-Siberian and Iranian-Turanian phytogeographic regions. 800 hectares of the area was forest land and 250 hectares of it was glades.

\section{Forest Vegetation}

Forest trees dominant at the park were in turn Pinus sylvestris, P.nigra, abies nordmanniana subsp. Bornmuelleriana, and Quercus pubescens. The ratios of the dominant forest trees according to Amenajman plan prepared in the area are as follows: 
$65 \%$ Pinus sylvestris

$24 \%$ P.nigra

$6 \%$ Abies nordmanniana subsp. bornmuelleriana

$5 \%$ Quercus pubescens

Moreover, the other trees found in the area were as follows:

Salix alba, S.caprea, Populus tremula, Carpinus betulus, Acer platanoides, A.campestre, A.hyrcanum, Sorbus umbellata, Crataegus monogyna, and Pyrus elaeagnifolia.

Some of the herbaceous species making up the under-wood vegetation were Polygala papilionacea, Vicia noeana, V. pannonice, Lathyrus pratensis, Campanula rapunculoides, and Orthilia secunda.

\section{Mountain and Pasture Vegetation}

Components of this vegetation exist at the glades and stream beds that dry up in summer. At the glades, Alyssum sibiricum, Astragalus plumosus, A. Micropterus, Hypericum heterophyllum, Acantholimon ulicinum, and Dipsacus laciniatus grow in abudance. At stream sides, Datisca cannabina, Lythrum salicaria, Lysimachia verticillaris, and Mentha longifolia species are common.

In addition to these common vegetation species, rock vegetation including abundant number of Crassulaceae family's genera (Sedum and Sempervivum) and Paronchia kurdica and Ephedra major grow at the rocks located in the area.

\section{MATERIAL AND METHOD}

The study materials were collected at 2-3 day trips made during the months of March through October in 1989-1990. The same regions were revisited during the study period at various vegetation stages and additional samples were collected for samples that were considered insufficient. 36 color slide films of plants that make up the area vegetation and topographic structure and some interesting plants were recorded.

The collected plant samples were pressed and dried according to the herbarium technique. Nearly all of the samples were named by benefiting from the book titled "Flora of Turkey and the East Aegean Islands". The species that were difficult to be named were characterized by using the book titled "Flora Europe, Flora of Iraq, the Genera of Flowering Plants". For some species that were difficult to characterize, the book titled "Botanical Latin, Vascular Plant Systematic" was used. The publications on A4 grid were benefited for determining species that were new for the grid. The named plant species were then categorized at family, genera and species levels and glued on cardboards. Furthermore, analogue samples, if any, were separated. The plants are preserved at the herbarium of Gazi University, Faculty of Arts and Sciences.

The sequencing used in the flora of Turkey was followed in the thesis to determine the order of families, genera and species.

First the genera name, then the species name and subspecies taxon name, if any, were given in turn when writing the plant list. Since the entire A4 grid is located within the limits of Ankara City, Kizılcahamam County, these information were not written to avoid repetition when writing the locality. The species list was prepared according to the evolution order like in the book of Davis titled "Flora of Turkey and the East Aegean Islands". 11 EUNIS Habitat codes were determined in the area and the situations of the plants were determined according to the phyto-geographic and the IUCN categories, and they are shown in the table in the flora list of the area. 
FLORA OF THE STUDY AREA

\begin{tabular}{|c|c|c|c|c|c|c|}
\hline No & Family & Species & $\begin{array}{c}\text { EUNIS } \\
\text { Habitat } \\
\text { code }\end{array}$ & Endemism & $\begin{array}{l}\text { Phytogeogra } \\
\text { Phical Region }\end{array}$ & $\begin{array}{c}\text { Endangerment } \\
\text { statue }\end{array}$ \\
\hline 1 & Equisetaceae & E.palustre L. & C2.6 & - & - & $\mathbf{L C}$ \\
\hline 2 & Polpodiaceae & Ceterach officinarum DC & C2.6 & - & - & LC \\
\hline 3 & Pinaceae & $\begin{array}{l}\text { Abies.nordmanniana } \\
\text { (Stev.) Spach.subsp. } \\
\text { bornmuelleriana (Mattf.) } \\
\text { Coode et Cullen } \\
\end{array}$ & G3.1 & Endemic & Oxine element & LC \\
\hline 4 & Pinaceae & Cedrus libani A.Rich & G3.4 & - & - & LC \\
\hline 5 & Pinaceae & Pinus.sylvestris L. & G4.5 & - & $\begin{array}{c}\text { Euro-Sib. } \\
\text { element }\end{array}$ & $\mathbf{L C}$ \\
\hline 6 & Pinaceae & $\begin{array}{l}\text { Pinus nigra Arn. subsp. } \\
\text { pallasiana (Lamb.) } \\
\text { Halmboe }\end{array}$ & G4.D & - & - & $\mathbf{L C}$ \\
\hline 7 & Cupressaaceae & $\begin{array}{l}\text { Juniperus communis L. } \\
\text { subsp. nana Syme }\end{array}$ & G4.9 & - & - & $\mathbf{L C}$ \\
\hline 8 & Cupressaaceae & $\begin{array}{c}\text { Juniperus oxycedrus L. } \\
\text { subsp. Oxycedrus }\end{array}$ & G4.9 & - & - & LC \\
\hline 9 & Cupressaaceae & Thuja orientalis L. & G3.4 & - & - & LC \\
\hline 10 & Ephedraceae & Ephedra major Host. & C2.6 & - & - & $\mathbf{L C}$ \\
\hline 11 & Ranunculaceae & $\begin{array}{l}\text { Nigella nigellastrum (L.) } \\
\text { Wilk. }\end{array}$ & C2.6 & - & - & LC \\
\hline 12 & Ranunculaceae & Ranunculus repens L. & C2.6 & - & Cosmopolitan & LC \\
\hline 13 & Ranunculaceae & $\begin{array}{l}\text { Ranunculus.constantinopo } \\
\text { litanus (DC.) d'Urv. }\end{array}$ & $\mathrm{C2.6}$ & - & Cosmopolitan & $\mathbf{L C}$ \\
\hline 14 & Ranunculaceae & $\begin{array}{l}\text { Ranunculus polyrhizos } \\
\text { Steph. }\end{array}$ & C2.6 & - & $\begin{array}{c}\text { Euro-Sib } \\
\text { element }\end{array}$ & $\mathbf{L C}$ \\
\hline 15 & Ranunculaceae & $\begin{array}{l}\text { Ranunculus damascenus } \\
\text { Boiss. Et. Gaill }\end{array}$ & G4.8 & - & Ir-Tur. Element & $\mathbf{L C}$ \\
\hline 16 & Ranunculaceae & $\begin{array}{c}\text { Ranunculus illyricus } \\
\text { L.subsp. illyricus }\end{array}$ & G4.8 & - & Cosmopolitan & $\mathbf{L C}$ \\
\hline 17 & Ranunculaceae & $\begin{array}{l}\text { Ceratocephalus falcatus } \\
\text { (L.) Pers. }\end{array}$ & G4.8 & - & Cosmopolitan & $\mathbf{L C}$ \\
\hline 18 & Berberidaceae & Berber1s crataegina DC. & G4.8 & - & Ir-Tur. Element & LC \\
\hline 19 & Papaveraceae & Chelıdonıum majus L. & C2.6 & - & $\begin{array}{c}\text { Euro-Sib. } \\
\text { element }\end{array}$ & LC \\
\hline 20 & Papaveraceae & $\begin{array}{l}\text { Papaver apokrinomenon } \\
\text { Fedde }\end{array}$ & G4-8 & Endemic & (1) & $\mathbf{L C}$ \\
\hline 21 & Papaveraceae & Papaver lacerum Popov. & G4.8 & - & Cosmopolitan & LC \\
\hline 22 & Papaveraceae & Papaver dubium L. & G4.8 & - & Cosmopolitan & LC \\
\hline 23 & Papaveraceae & $\begin{array}{c}\text { Corydalıs solida Swartz } \\
\text { subsp. Solida }\end{array}$ & G4.8 & - & Cosmopolitan & LC \\
\hline 24 & Papaveraceae & Fumaria cilicica Hausskn & G4.8 & - & Ir-Tur. element & LC \\
\hline 25 & Papaveraceae & Fumaria asepala Boiss. & G4.8 & - & Ir-Tur. element & LC \\
\hline 26 & Brassicaceae & $\begin{array}{l}\text { Cardaria draba Desv. } \\
\text { subsp. Draba }\end{array}$ & G1.B & - & Cosmopolitan & $\mathbf{L C}$ \\
\hline 27 & Brassicaceae & $\begin{array}{l}\text { Isat1s cappadocica Desv. } \\
\text { subsp.cappadocica }\end{array}$ & G4.D & - & Ir-Tur. element & LC \\
\hline 28 & Brassicaceae & $\begin{array}{l}\text { Isatis cappadocica Desv. } \\
\text { subsp. alyssifolia (Boiss.) } \\
\text { Davis }\end{array}$ & G4.D & - & Ir-Tur. element & LC \\
\hline 29 & Brassicaceae & $\begin{array}{l}\text { Aethionema arabicum (L) } \\
\text { Andrz. }\end{array}$ & G4.D & - & Ir-Tur. element & LC \\
\hline 30 & Brassicaceae & $\begin{array}{l}\text { Capsella } \\
\text { bursa-pastoris (L) Medik. }\end{array}$ & G4.D & - & Cosmopolitan & $\mathbf{L C}$ \\
\hline 31 & Brassicaceae & $\begin{array}{l}\text { Fibıgıa clypeata (L) } \\
\text { Medik. }\end{array}$ & G4.D & - & Cosmopolitan & LC \\
\hline 32 & Brassicaceae & $\begin{array}{l}\text { Fibıgıa eriocarpa (DC) } \\
\text { Boiss. }\end{array}$ & G4.D & - & Cosmopolitan & $\mathbf{L C}$ \\
\hline
\end{tabular}


International Journal of Scientific and Technological Research

ISSN 2422-8702 (Online), DOI: 10.7176/JSTR/5-2-43

Vol.5, No.2, 2019

\begin{tabular}{|c|c|c|c|c|c|c|}
\hline 33 & Brassicaceae & $\begin{array}{l}\text { Alyssum linifolium Steph. } \\
\text { var linifolium }\end{array}$ & G4.D & - & Cosmopolitan & LC \\
\hline 34 & Brassicaceae & $\begin{array}{l}\text { Alyssum minutum } \\
\text { Schlecht. }\end{array}$ & G4.D & - & Cosmopolitan & LC \\
\hline 35 & Brassicaceae & $\begin{array}{l}\text { Alyssum strigosum Banks } \\
\text { et. subsp. Strigosum }\end{array}$ & G4.D & - & Cosmopolitan & $\mathbf{L C}$ \\
\hline 36 & Brassicaceae & $\begin{array}{l}\text { Alyssum xanthovarpum } \\
\text { Boiss. } \\
\end{array}$ & G4.D & - & Cosmopolitan & LC \\
\hline 37 & Brassicaceae & Alyssum sibiricum Willd. & G4.D & - & Cosmopolitan & LC \\
\hline 38 & Brassicaceae & $\begin{array}{c}\text { A.murale Waldst. Et Kit } \\
\text { subsp. murale var. Murale }\end{array}$ & G4.D & - & Cosmopolitan & $\mathbf{L C}$ \\
\hline 39 & Brassicaceae & $\begin{array}{l}\text { Arabis sagittata (Bertol.) } \\
\text { DC. }\end{array}$ & G4.D & - & Cosmopolitan & $\mathbf{L C}$ \\
\hline 40 & Brassicaceae & Turritıs glabra L. & G4.D & - & Cosmopolitan & LC \\
\hline 41 & Brassicaceae & $\begin{array}{l}\text { Turritıs laxa (Sibth. Et } \\
\text { Sm.) Hayek }\end{array}$ & G4.D & - & Cosmopolitan & $\mathbf{L C}$ \\
\hline 42 & Brassicaceae & $\begin{array}{l}\text { Barbarea trichopoda } \\
\text { Hausskn. }\end{array}$ & C2.6 & Endemic & - & LC \\
\hline 43 & Brassicaceae & $\begin{array}{l}\text { Hesperis bicuspidata } \\
\text { (Wild.) Poiret }\end{array}$ & G4.D & - & Cosmopolitan & $\mathbf{L C}$ \\
\hline 44 & Brassicaceae & $\begin{array}{l}\text { Erysımum cuspidatum } \\
\text { (Bieb.) DC. }\end{array}$ & G3.4 & - & Cosmopolitan & LC \\
\hline 45 & Brassicaceae & $\begin{array}{c}\text { Erysımum eginense } \\
\text { Hausskn. Ex Bornm. }\end{array}$ & G3.4 & Endemic & - & $\mathbf{V U}$ \\
\hline 46 & Brassicaceae & $\begin{array}{c}\text { Erysimum smyrnaeum } \\
\text { Boiss. et Bal. }\end{array}$ & G4.D & - & Cosmopolitan & LC \\
\hline 47 & Brassicaceae & $\begin{array}{l}\text { Alliaria petiolata (Bieb.) } \\
\text { Cavara et Grande } \\
\end{array}$ & G4.D & - & Cosmopolitan & $\mathbf{L C}$ \\
\hline 48 & Brassicaceae & Sisymbrium altissimum L. & G4.D & - & Cosmopolitan & LC \\
\hline 49 & Brassicaceae & $\begin{array}{l}\text { Descuraınıa sophia (L.) } \\
\text { Webb }\end{array}$ & G1.B & - & Cosmopolitan & LC \\
\hline 50 & Resedaceae & Reseda lutea L. Var lutea & G4.D & - & Cosmopolitan & LC \\
\hline 51 & Cistaceae & Cistus laurifolius L. & G4.9 & - & Mediterranean & LC \\
\hline 52 & Cistaceae & $\begin{array}{l}\text { Helianthemum } \\
\text { nummularium (L.) Miller } \\
\text { subsp. lycaonicum Coode } \\
\text { et Cullen }\end{array}$ & G4.9 & Endemic & - & $\mathbf{L C}$ \\
\hline 53 & Cistaceae & $\begin{array}{l}\text { Helianthemum ledifolium } \\
\text { (L.) Miller var. } \\
\text { Ledifolium }\end{array}$ & G3.9 & - & Cosmopolitan & LC \\
\hline 54 & Violaceae & Viola odorata L. & C2.6 & - & Cosmopolitan & LC \\
\hline 55 & Violaceae & Viola sieheana Becker & G4.9 & - & Cosmopolitan & LC \\
\hline 56 & Violaceae & Viola occulta Lehm. & G4.9 & - & Cosmopolitan & $\mathbf{L C}$ \\
\hline 57 & Violaceae & $\begin{array}{l}\text { Viola gracilis Sibth. Et } \\
\text { Sm. }\end{array}$ & G4.9 & - & Cosmopolitan & LC \\
\hline 58 & Polygalaceae & $\begin{array}{l}\text { Polygala papilionacea } \\
\text { Boiss. }\end{array}$ & G4.D & - & Ir-Tur. element & LC \\
\hline 59 & Caryophyllaceae & $\begin{array}{l}\text { Arenarı ledebouriana } \\
\text { Fenzl. var. Ledebouriana }\end{array}$ & G4.7 & Endemic & - & LC \\
\hline 60 & Caryophyllaceae & $\begin{array}{l}\text { Minuartıa hirsuta (Bieb.) } \\
\text { Hand.-Mazz. subsp. } \\
\text { falcata (Gris.) Mattf. }\end{array}$ & G4.D & - & Cosmopolitan & LC \\
\hline 61 & Caryophyllaceae & $\begin{array}{l}\text { Minuartıa hamata } \\
\text { (Hausskn.) Mattf. }\end{array}$ & G4.D & - & Cosmopolitan & LC \\
\hline 62 & Caryophyllaceae & $\begin{array}{l}\text { Stellaria media (L.) Vill. } \\
\text { subsp. media }\end{array}$ & C2.6 & - & Cosmopolitan & LC \\
\hline 63 & Caryophyllaceae & $\begin{array}{l}\text { Cerastıum dichotomum L. } \\
\text { Subsp. Dichotomum }\end{array}$ & G4.D & - & - & LC \\
\hline
\end{tabular}


Vol.5, No.2, 2019

\begin{tabular}{|c|c|c|c|c|c|c|}
\hline & & $\begin{array}{l}\text { Cerastium brachypetalum } \\
\text { Pers. Subsp. Roeseri } \\
\text { (Boiss. Et Heldr.) Nyman }\end{array}$ & & & & \\
\hline 64 & Caryophyllaceae & Cerastıum. gracile Duf & G4.D & - & Cosmopolitan & LC \\
\hline 65 & Caryophyllaceae & $\begin{array}{l}\text { Moenchia mantica (L.) } \\
\text { Bartl. subsp. Mantica }\end{array}$ & G4.D & - & Cosmopolitan & LC \\
\hline 66 & Caryophyllaceae & $\begin{array}{l}\text { Telephium imperati L. } \\
\text { subsp. orientale (Boiss.) } \\
\text { Nyman }\end{array}$ & G4.D & - & Cosmopolitan & $\mathbf{L C}$ \\
\hline 67 & Caryophyllaceae & $\begin{array}{l}\text { Dianthus micranthus } \\
\text { Boiss. Et Heldr. }\end{array}$ & G4.D & - & Cosmopolitan & LC \\
\hline 68 & Caryophyllaceae & $\begin{array}{l}\text { Dianthus ancyrensis } \\
\text { Hausskn. Et Bornm. }\end{array}$ & G4.5 & Endemic & Ir.-Tur.element & $\mathbf{V U}$ \\
\hline 69 & Caryophyllaceae & $\begin{array}{c}\text { Dianthus zonatus Fenzl. } \\
\text { Var. Aristatus (Boiss.) } \\
\text { Reeve } \\
\end{array}$ & G4.5 & - & - & LC \\
\hline 70 & Caryophyllaceae & Dianthus lydus Boiss. & G4.7 & Endemic & - & VU \\
\hline 71 & Caryophyllaceae & $\begin{array}{l}\text { Petrorhagia alpina (Habl.) } \\
\text { Ball et Heywood subsp. } \\
\text { Alpina }\end{array}$ & G4.5 & - & - & $\mathbf{L C}$ \\
\hline 72 & Caryophyllaceae & $\begin{array}{l}\text { Saponaria chlorifolia } \\
\text { Kunze }\end{array}$ & G4.D & Endemic & - & $\mathbf{V U}$ \\
\hline 73 & Caryophyllaceae & Silene italica (L.) Pers. & G4.D & - & Cosmopolitan & LC \\
\hline 74 & Caryophyllaceae & Silene chlorifolia Sm. & G4.D & - & Ir.-Tur.element & $\mathbf{L C}$ \\
\hline 75 & Caryophyllaceae & $\begin{array}{l}\text { Silene supina Bieb. subsp. } \\
\text { Pruinosa (Boiss.) } \\
\text { Chowdh. }\end{array}$ & G3.4 & - & - & $\mathbf{L C}$ \\
\hline 76 & Caryophyllaceae & $\begin{array}{l}\text { Silene vulgaris (Moench) } \\
\text { Garcke var. Vulgaris }\end{array}$ & G4.5 & - & - & $\mathbf{L C}$ \\
\hline 77 & Caryophyllaceae & $\begin{array}{l}\text { Silene fabaria (L.) Sibth. } \\
\text { Et Sm. }\end{array}$ & G4.5 & - & - & $\mathbf{L C}$ \\
\hline 78 & Caryophyllaceae & Silene compacta Fischer & G4.5 & - & Cosmopolitan & $\mathbf{L C}$ \\
\hline 79 & Caryophyllaceae & \begin{tabular}{|l|} 
Silene alba (Miller) \\
Krause subsp. eriocalycina \\
(Boiss.) Walter
\end{tabular} & G4.D & - & - & LC \\
\hline 80 & Caryophyllaceae & $\begin{array}{l}\text { Silene dichotoma Ehrh. } \\
\text { subsp. sibthorpiana } \\
\text { (Reichb.) Rech. }\end{array}$ & G4.D & - & Cosmopolitan & LC \\
\hline 81 & İllecebraceae & $\begin{array}{l}\text { Herniaria micrantha A.K } \\
\text { Jackson et Turrill }\end{array}$ & G4.9 & - & Mediterranean & $\mathbf{L C}$ \\
\hline 82 & İllecebraceae & Hernıarıa incana Lam. & G4.5 & - & Cosmopolitan & $\mathbf{L C}$ \\
\hline 83 & İllecebraceae & $\begin{array}{l}\text { Paronychia kurdica Boiss. } \\
\text { subsp. kurdica var. } \\
\text { Kurdica }\end{array}$ & G4.D & - & - & LC \\
\hline 84 & İllecebraceae & $\begin{array}{l}\text { Scleranthus uncinatus } \\
\text { Schur. }\end{array}$ & G4.D & - & Cosmopolitan & LC \\
\hline 85 & Polygonaceae & $\begin{array}{l}\text { Polygonum lapathifolium } \\
\text { L. }\end{array}$ & C2.6 & - & - & $\mathbf{L C}$ \\
\hline 86 & Polygonaceae & $\begin{array}{c}\text { Polygonum cognatum } \\
\text { Meissn. }\end{array}$ & G3.4 & - & Cosmopolitan & $\mathbf{L C}$ \\
\hline 87 & Polygonaceae & Polygonum bellardii All. & C2.6 & - & Cosmopolitan & $\mathbf{L C}$ \\
\hline 88 & Polygonaceae & Rumex acetosella L. & G4.5 & - & Cosmopolitan & $\mathbf{L C}$ \\
\hline 89 & Polygonaceae & Rumex scutatus L. & G4.D & - & Cosmopolitan & $\mathbf{L C}$ \\
\hline 90 & Polygonaceae & Rumex crispus L. & G4.5 & - & - & $\mathbf{L C}$ \\
\hline 91 & Chenopodiaceae & Chenopodium botrys L & C2.6 & - & - & $\mathbf{L C}$ \\
\hline 92 & Chenopodiaceae & $\begin{array}{l}\text { Chenopodium foliosum } \\
\text { (Moench), Aschers }\end{array}$ & C2.6 & - & Cosmopolitan & $\mathbf{L C}$ \\
\hline 93 & Chenopodiaceae & \begin{tabular}{|} 
Chenopodium 382album \\
L. subsp. 382album var. \\
Albüm
\end{tabular} & C2.6 & - & - & LC \\
\hline 94 & Elatınaceae & Elatıne alsinastrum L. & G3.4 & - & - & $\mathbf{L C}$ \\
\hline 95 & Hypericaceae & $\begin{array}{l}\text { Hypericum heterophyllum } \\
\text { Vent. }\end{array}$ & G4.D & - & Endemic & $\mathbf{L C}$ \\
\hline
\end{tabular}


Vol.5, No.2, 2019

\begin{tabular}{|c|c|c|c|c|c|c|}
\hline 96 & Hypericaceae & Hypericum scabrum L. & G4.D & - & Ir.-Tur.element & $\mathbf{L C}$ \\
\hline 97 & Hypericaceae & Hypericum orientale L. & G3.4 & - & - & LC \\
\hline 98 & Hypericaceae & Hypericum perforatum L. & G3.4 & - & Cosmopolitan & $\mathbf{L C}$ \\
\hline 99 & Malvaceae & Malva alcea L. & G3.4 & - & - & LC \\
\hline 100 & Malvaceae & $\begin{array}{l}\text { Alcea pallida Waldst. et } \\
\text { Kit }\end{array}$ & G3.4 & - & - & $\mathbf{L C}$ \\
\hline 101 & Tiliaceae & Tilia cordata. Miller & C2.6 & - & - & $\mathbf{L C}$ \\
\hline 102 & Geraniaceae & Geranium robertianum L & G4.D & - & - & LC \\
\hline 103 & Geraniaceae & $\begin{array}{l}\text { Geranium macrostylum } \\
\text { Boiss. }\end{array}$ & G4.D & - & $\begin{array}{c}\text { Mediterreanean } \\
\text { mountain }\end{array}$ & LC \\
\hline 104 & Geraniaceae & $\begin{array}{l}\text { Geranıum pyrenaicum } \\
\text { Burm. fil }\end{array}$ & G4.D & - & - & LC \\
\hline 105 & Geraniaceae & $\begin{array}{l}\text { Erodium acaule (L.) } \\
\text { Becherer \& Thell. }\end{array}$ & G4.D & - & Mediterreanean & $\mathbf{L C}$ \\
\hline 106 & Geraniaceae & $\begin{array}{l}\text { Pelargonium } \\
\text { endlicherianum Fenzl. }\end{array}$ & G4.D & - & Cosmopolitan & LC \\
\hline 107 & Aceraceae & Acer platanoides L. & G4.8 & - & $\begin{array}{c}\text { Euro- } \\
\text { Sib.element } \\
\end{array}$ & $\mathbf{L C}$ \\
\hline 108 & Aceraceae & $\begin{array}{l}\text { Acer campestre L. subsp. } \\
\text { Campestre }\end{array}$ & G4.8 & - & - & $\mathbf{L C}$ \\
\hline 109 & Aceraceae & $\begin{array}{l}\text { Acer hyrcanum Frsch. et } \\
\text { Mey. subsp. Hyrcanum }\end{array}$ & G4.8 & - & $\begin{array}{c}\text { Euro- } \\
\text { Sib.element }\end{array}$ & LC \\
\hline 110 & Aceraceae & Acer negundo L. & C2.6 & - & - & $\mathbf{L C}$ \\
\hline 111 & Vitaceae & Vtıs sylvestris Gmelin & G4.8 & - & Cosmopolitan & $\mathbf{L C}$ \\
\hline 112 & Anacardiaceae & $\begin{array}{l}\text { Pistacia terebinthus L. } \\
\text { subsp. palaestina (Boiss.) } \\
\text { Engler }\end{array}$ & G4.D & - & - & $\mathbf{L C}$ \\
\hline 113 & Celastraceae & $\begin{array}{l}\text { Euonymus latifolius (L.) } \\
\text { Miller subsp. cauconis } \\
\text { Coode et Cullen }\end{array}$ & G4.8 & - & - & $\mathbf{V U}$ \\
\hline 114 & Celastraceae & Euonymus europaeus L. & G4.D & - & $\begin{array}{c}\text { Euro- } \\
\text { Sib.element }\end{array}$ & $\mathbf{L C}$ \\
\hline 115 & Fabaceae & Sophora japonica L. & C2.6 & - & - & LC \\
\hline 116 & Fabaceae & $\begin{array}{l}\text { Chamaecytisus pygmaeus } \\
\text { (Willd.) Rothm. }\end{array}$ & G3.4 & - & $\begin{array}{c}\text { Euro- } \\
\text { Sib.element }\end{array}$ & $\mathbf{L C}$ \\
\hline 117 & Fabaceae & Robınıa pseudoacacia L. & G4.D & - & - & $\mathbf{L C}$ \\
\hline 118 & Fabaceae & Galega officinalis L. & G4.7 & - & $\begin{array}{c}\text { Euro- } \\
\text { Sib.element }\end{array}$ & $\mathbf{L C}$ \\
\hline 119 & Fabaceae & $\begin{array}{l}\text { Colutea cilicica Boiss. \& } \\
\text { BaL. }\end{array}$ & G4.7 & - & - & $\mathbf{L C}$ \\
\hline 120 & Fabaceae & $\begin{array}{l}\text { Astragalus coodei Chamb. } \\
\text { \& Matthews }\end{array}$ & G4.7 & Endemic & - & LC \\
\hline 121 & Fabaceae & $\begin{array}{l}\text { Astragalus glycyphyllos } \\
\text { L. subsp. glycyphylloides } \\
\text { (DC) Matthews }\end{array}$ & G3.4 & - & $\begin{array}{c}\text { Euro- } \\
\text { Sib.element }\end{array}$ & $\mathbf{L C}$ \\
\hline 122 & Fabaceae & $\begin{array}{l}\text { Astragalus plumosus } \\
\text { Willd. Var. Plumosus }\end{array}$ & G3.4 & - & - & $\mathbf{L C}$ \\
\hline 123 & Fabaceae & $\begin{array}{l}\text { Astragalus micropterus } \\
\text { Fischer }\end{array}$ & G3.4 & - & Ir.-Tur.element & $\mathbf{L C}$ \\
\hline 124 & Fabaceae & $\begin{array}{l}\text { Astragalus brachypterus } \\
\text { Fischer }\end{array}$ & G4.F & Endemic & Ir.-Tur.element & $\mathbf{L C}$ \\
\hline 125 & Fabaceae & $\begin{array}{c}\text { Astragalus mitchelianus } \\
\text { Boiss. }\end{array}$ & G4.F & Endemic & - & LC \\
\hline 126 & Fabaceae & Cicer anatolicum Alet. & G4.D & - & Ir.-Tur.element & $\mathbf{L C}$ \\
\hline 127 & Fabaceae & $\begin{array}{l}\text { Vicia cracca L. subsp. } \\
\text { stenophylla Yel. }\end{array}$ & G4.5 & - & - & $\mathbf{L C}$ \\
\hline 128 & Fabaceae & $\begin{array}{l}\text { Vicia monantha Retz. } \\
\text { subsp. Monantha }\end{array}$ & G4.D & - & - & LC \\
\hline 129 & Fabaceae & $\begin{array}{l}\text { Vicia caesarea Boiss. Et } \\
\text { Bal. }\end{array}$ & G4.D & Endemic & Ir.-Tur.element & $\mathbf{L C}$ \\
\hline 130 & Fabaceae & $\begin{array}{l}\text { Vicia hirsuta (L.) S.F. } \\
\text { Gray }\end{array}$ & G4.D & - & - & LC \\
\hline
\end{tabular}


Vol.5, No.2, 2019

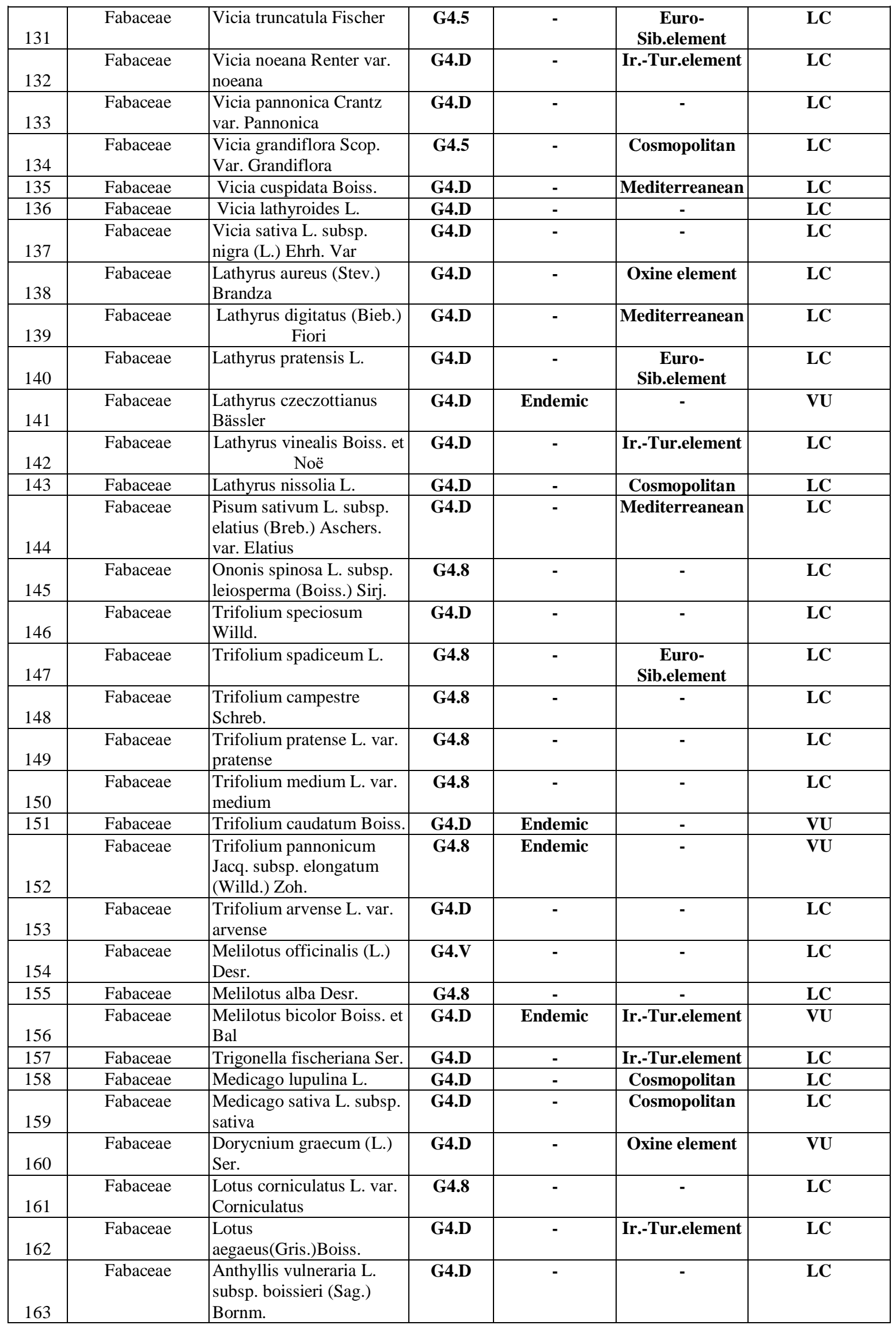


Vol.5, No.2, 2019

\begin{tabular}{|c|c|c|c|c|c|c|}
\hline 164 & Fabaceae & \begin{tabular}{|l} 
Coronilla varia L. subsp. \\
Varia \\
\end{tabular} & G4.D & - & Cosmopolitan & LC \\
\hline 165 & Rosaceae & $\begin{array}{l}\text { Prunus divaricata. Ledeb. } \\
\text { subsp. Divaricata }\end{array}$ & G4.D & - & - & $\mathbf{L C}$ \\
\hline 166 & Rosaceae & $\begin{array}{l}\text { Cerasus avium (L.) } \\
\text { Moench }\end{array}$ & G3.4 & - & - & $\mathbf{L C}$ \\
\hline 167 & Rosaceae & $\begin{array}{l}\text { Cerasus mahaleb (L.) } \\
\text { Miller var. Mahaleb } \\
\end{array}$ & G3.4 & - & - & LC \\
\hline 168 & Rosaceae & Armeniaca vulgaris Lam. & G3.4 & - & - & LC \\
\hline 169 & Rosaceae & Rubus idaeus L. & G3.4 & - & - & $\mathbf{L C}$ \\
\hline 170 & Rosaceae & $\begin{array}{l}\text { Rubus discolor Weihe et } \\
\text { Nees }\end{array}$ & G4.D & - & - & $\mathbf{L C}$ \\
\hline 171 & Rosaceae & $\begin{array}{l}\text { Rubus canescens DC. var. } \\
\text { Canescens }\end{array}$ & G4.D & - & - & LC \\
\hline 172 & Rosaceae & Potentilla rupestris L. & G3.1 & - & $\begin{array}{c}\text { Euro.Sib.eleme } \\
\text { nt }\end{array}$ & LC \\
\hline 173 & Rosaceae & Potentilla recta L. & G3.1 & - & Cosmopolitan & LC \\
\hline 174 & Rosaceae & Fragaria vesca L. & G3.1 & - & - & LC \\
\hline 175 & Rosaceae & Geum urbanum L. & G3.1 & - & $\begin{array}{c}\text { Euro- } \\
\text { Sib.element }\end{array}$ & LC \\
\hline 176 & Rosaceae & Agrimonia eupatoria L. & G3.4 & - & - & LC \\
\hline 177 & Rosaceae & $\begin{array}{l}\text { Sanguisorba minor Scop. } \\
\text { subsp. muricata (Spach) } \\
\text { Briq. }\end{array}$ & G4.5 & - & - & $\mathbf{L C}$ \\
\hline 178 & Rosaceae & $\begin{array}{l}\text { Alchemilla mollis (Buser) } \\
\text { Rothm. }\end{array}$ & G4.5 & - & Cosmopolitan & $\mathbf{L C}$ \\
\hline 179 & Rosaceae & Rosa canina L. & G4.D & - & - & $\mathbf{L C}$ \\
\hline 180 & Rosaceae & $\begin{array}{l}\text { Cotoneaster nummularia } \\
\text { Fisch. et Mey. }\end{array}$ & G4.5 & - & Cosmopolitan & $\mathbf{L C}$ \\
\hline 181 & Rosaceae & $\begin{array}{l}\text { Crataegus pentagyna } \\
\text { Waldst et Kit }\end{array}$ & G4.8 & - & $\begin{array}{c}\text { Euro.Sib.eleme } \\
\text { nt }\end{array}$ & LC \\
\hline 182 & Rosaceae & $\begin{array}{l}\text { Crataegus x bornmuelleri } \\
\text { Zabel }\end{array}$ & G4.D & Endemic & - & LC \\
\hline 183 & Rosaceae & $\begin{array}{l}\text { Crataegus monogyna Jacq. } \\
\text { subsp. Monogyna }\end{array}$ & G4.D & - & - & $\mathbf{L C}$ \\
\hline 184 & Rosaceae & $\begin{array}{l}\text { Sorbus umbellata (Desf.) } \\
\text { Fritsch var. Umbellata } \\
\end{array}$ & G4.5 & - & - & LC \\
\hline 185 & Rosaceae & $\begin{array}{l}\text { Sorbus torminalis (L.) } \\
\text { Crantz var. Torminalis }\end{array}$ & G4.5 & - & - & LC \\
\hline 186 & Rosaceae & $\begin{array}{l}\text { Malus sylvestris Miller } \\
\text { subsp. orientalis } \\
\text { (A.Uglitzleich) }\end{array}$ & G3.4 & - & - & LC \\
\hline 187 & Rosaceae & $\begin{array}{l}\text { Pyrus communis L. subsp. } \\
\text { sativa (DC.) Hegi }\end{array}$ & G3.4 & - & - & $\mathbf{L C}$ \\
\hline 188 & Rosaceae & $\begin{array}{l}\text { Pyrus elaeagnifolia Pallas } \\
\text { subsp. Elaeagnifolia }\end{array}$ & G4.D & - & - & $\mathbf{L C}$ \\
\hline 189 & L ythraceae & Lythrum salicaria L. & C2.6 & - & $\begin{array}{c}\text { Euro.Sib.eleme } \\
\text { nt }\end{array}$ & $\mathbf{L C}$ \\
\hline 190 & Onagraceae & $\begin{array}{l}\text { Epilobium angustifolium } \\
\text { L. }\end{array}$ & G4.8 & - & - & LC \\
\hline 191 & Onagraceae & Epilobium hirsutum L. & G4.8 & - & Cosmopolitan & LC \\
\hline 192 & Onagraceae & $\begin{array}{l}\text { Epilobium lanceolatum } \\
\text { Seb. et MaurI }\end{array}$ & G4.8 & - & - & $\mathbf{L C}$ \\
\hline 193 & Cucurbitaceae & Bryonia alba. L. & C2.6 & - & $\begin{array}{c}\text { Euro.Sib.eleme } \\
\text { nt }\end{array}$ & LC \\
\hline 194 & Datiscaceae & Datisca cannabina L. & G4.D & - & - & LC \\
\hline 195 & Crassulaceae & Umbilicus erectus DC. & H3.6 & - & Cosmopolitan & LC \\
\hline 196 & Crassulaceae & \begin{tabular}{|l|}
$\begin{array}{l}\text { Sedum obtusifolium C.A. } \\
\text { Meyer }\end{array}$ \\
\end{tabular} & H3.6 & - & - & LC \\
\hline 197 & Crassulaceae & Sedum amplexicaule DC & H3.6 & - & Mediterreanean & $\mathbf{L C}$ \\
\hline 198 & Crassulaceae & Sedum album L. & H3.6 & - & - & $\mathbf{L C}$ \\
\hline 199 & Crassulaceae & $\begin{array}{l}\text { Sedum subulatum } \\
\text { (C.A.Meyer) Boiss. }\end{array}$ & H3.6 & - & - & LC \\
\hline
\end{tabular}


Vol.5, No.2, 2019

\begin{tabular}{|c|c|c|c|c|c|c|}
\hline 200 & Crassulaceae & $\begin{array}{c}\text { Sedum sempervivoides } \\
\text { Bieb. }\end{array}$ & H3.6 & - & - & LC \\
\hline 201 & Crassulaceae & $\begin{array}{l}\text { Sedum caespitosum (Cav.) } \\
\text { DC. }\end{array}$ & H3.6 & - & Mediterreanean & LC \\
\hline 202 & Crassulaceae & $\begin{array}{l}\text { Sedum hispanicum L. var. } \\
\text { Hispanicum }\end{array}$ & G3.4 & - & Cosmopolitan & LC \\
\hline 203 & Crassulaceae & $\begin{array}{l}\text { Sedum pallidum Bieb. var. } \\
\text { Pallidum }\end{array}$ & G4.D & - & Cosmopolitan & LC \\
\hline 204 & Crassulaceae & $\begin{array}{l}\text { Sedum pallidum Bieb. var. } \\
\text { Bithynicum (boiss.) } \\
\text { Chamberlain }\end{array}$ & G3.4 & - & Oxine element & LC \\
\hline 205 & Crassulaceae & $\begin{array}{l}\text { Senpervivum armenum } \\
\text { Boiss. et Huet var. insigne } \\
\text { Muirhead }\end{array}$ & H3.6 & Endemic & $\begin{array}{c}\text { Oxine Mountain } \\
\text { element }\end{array}$ & LC \\
\hline 206 & Saxifragaceae & $\begin{array}{l}\text { Saxifraga cymbalaria L. } \\
\text { var. Cymbalaria }\end{array}$ & C2.6 & - & Cosmopolitan & LC \\
\hline 207 & Apiaceae & $\begin{array}{l}\text { Eryngium campestre L. } \\
\text { var. virens Link. }\end{array}$ & G4.D & - & Cosmopolitan & LC \\
\hline 208 & Apiaceae & $\begin{array}{l}\text { Anthriscus nemorosa } \\
\text { (Bieb.) Sprengel }\end{array}$ & G4.D & - & Cosmopolitan & LC \\
\hline 209 & Apiaceae & Scandıx iberica Bieb. & G4.D & - & Cosmopolitan & LC \\
\hline 210 & Apiaceae & Scandix pecten-veneris L. & G4.7 & - & Cosmopolitan & LC \\
\hline 211 & Apiaceae & $\begin{array}{l}\text { Pımpınella tragium Will. } \\
\text { subsp. polyclada. (Boiss. } \\
\text { et Heldr.) Tutin }\end{array}$ & H3.6 & - & Cosmopolitan & LC \\
\hline 212 & Apiaceae & $\begin{array}{l}\text { P1mpınella tragium } \\
\text { Will.Subspecies } \\
\text { lithophila(Schischkin) } \\
\text { Tutin }\end{array}$ & G4.7 & - & - & LC \\
\hline 213 & Apiaceae & $\begin{array}{l}\text { Seselı peucedanoides } \\
\text { (Bieb.) Koso-Pol. }\end{array}$ & G4.7 & - & $\begin{array}{c}\text { Euro- } \\
\text { Sib.element }\end{array}$ & LC \\
\hline 214 & Apiaceae & Oenanthe silaifolia Bieb. & G4.7 & - & Cosmopolitan & LC \\
\hline 215 & Apiaceae & Conıum maculatum L. & G4.D & - & - & LC \\
\hline 216 & Apiaceae & \begin{tabular}{|l|} 
Prangos meliocarpoides \\
Boiss. var. Meliocarpoides
\end{tabular} & G4.D & Endemic & Ir.-Tur.element & LC \\
\hline 217 & Apiaceae & Bupleurum affiine Sadler & G4.D & - & - & LC \\
\hline 218 & Apiaceae & Bupleurum gerardii All. & G4.D & - & Cosmopolitan & LC \\
\hline 219 & Apiaceae & $\begin{array}{l}\text { Apıum nodiflorum (L.) } \\
\text { Lag. }\end{array}$ & C2.6 & - & Cosmopolitan & LC \\
\hline 220 & Apiaceae & $\begin{array}{l}\text { Petroselınum crispum } \\
\text { (Miller) A.W.Hill }\end{array}$ & C2.6 & - & - & LC \\
\hline 221 & Apiaceae & Falcarıa vulgaris Bernh. & G4.D & - & Cosmopolitan & LC \\
\hline 222 & Apiaceae & $\begin{array}{l}\text { Ferulago galbanifera } \\
\text { (Miller) W.Koch }\end{array}$ & H3.6 & - & $\begin{array}{c}\text { Euro- } \\
\text { Sib.element }\end{array}$ & LC \\
\hline 223 & Apiaceae & $\begin{array}{l}\text { Pastinaca sativa L. subsp. } \\
\text { urens (Req. ex Godron) } \\
\text { Celak }\end{array}$ & C2.6 & - & Cosmopolitan & LC \\
\hline 224 & Apiaceae & $\begin{array}{l}\text { Heracleum sphondylium } \\
\text { L. subsp. ternatum } \\
\text { (Velen.) Brummitt. }\end{array}$ & $\mathrm{C2.6}$ & - & $\begin{array}{c}\text { Euro- } \\
\text { Sib.element }\end{array}$ & LC \\
\hline 225 & Apiaceae & $\begin{array}{l}\text { Heracleum platytaenium } \\
\text { Boiss. }\end{array}$ & G4.D & Endemic & Oxine element & VU \\
\hline 226 & Apiaceae & $\begin{array}{l}\text { Laserpitium hispidum } \\
\text { Bieb. }\end{array}$ & G4.5 & - & $\begin{array}{c}\text { Euro- } \\
\text { Sib.element }\end{array}$ & LC \\
\hline 227 & Apiaceae & Torilis ucranica Sprengel & G4.D & - & - & LC \\
\hline 228 & Apiaceae & $\begin{array}{l}\text { Astrodaucus orientalis (L.) } \\
\text { Drude }\end{array}$ & G4.D & - & Ir.-Tur.element & LC \\
\hline 229 & Apiaceae & Caucalis platycarpos L. & G4.D & - & Cosmopolitan & LC \\
\hline 230 & Apiaceae & Daucus carota L. Grup C. & $\mathrm{C2.6}$ & - & - & LC \\
\hline 231 & Araliaceae & Hedera helix L. & C2.6 & - & - & LC \\
\hline
\end{tabular}


Vol.5, No.2, 2019

\begin{tabular}{|c|c|c|c|c|c|c|}
\hline 232 & Cornaceae & $\begin{array}{l}\text { Cornus sanguinea L. } \\
\text { subsp. australis (C.A. } \\
\text { Meyer) Jàv. }\end{array}$ & G4.D & - & $\begin{array}{c}\text { Euro- } \\
\text { Sib.element }\end{array}$ & LC \\
\hline 233 & Caprifoliaceae & Viburnum lantana L. & C2.6 & - & $\begin{array}{c}\text { Euro- } \\
\text { Sib.element }\end{array}$ & $\mathbf{L C}$ \\
\hline 234 & Caprifoliaceae & \begin{tabular}{|l|} 
Lonicera caucasica Pallas \\
subsp. orientalis (Lam.) \\
Chamb. et Long.
\end{tabular} & G4.5 & Endemic & - & $\mathbf{V U}$ \\
\hline 235 & Caprifoliaceae & $\begin{array}{l}\text { Lonicera etrusca Santr } \\
\text { var. Etrusca }\end{array}$ & G4.D & - & Mediterreanean & LC \\
\hline 236 & Valerianaceae & $\begin{array}{l}\text { Valeriana alliariifolia } \\
\text { Adams }\end{array}$ & C2.6 & - & - & $\mathbf{L C}$ \\
\hline 237 & Valerianaceae & Valeriana tuberosa L. & G4.D & - & - & LC \\
\hline 238 & Valerianaceae & $\begin{array}{l}\text { Centranthus longiflorus } \\
\text { Stev. subsp. Langiflorus }\end{array}$ & $\mathrm{C2.6}$ & - & Ir.-Tur.element & $\mathbf{L C}$ \\
\hline 239 & Valerianaceae & $\begin{array}{l}\text { Valerianella carinata. } \\
\text { Lois. }\end{array}$ & G4.D & - & Cosmopolitan & LC \\
\hline 240 & Valerianaceae & $\begin{array}{l}\text { Valerianella coronata (L.) } \\
\text { DC. }\end{array}$ & G4.D & - & Cosmopolitian & LC \\
\hline 241 & Dipsacaceae & Dipsacus laciniatus L. & G4.5 & - & Cosmopolitian & LC \\
\hline 242 & Dipsacaceae & Scabiosa argentea L. & G4.D & - & Cosmopolitian & $\mathbf{L C}$ \\
\hline 243 & Dipsacaceae & Scabiosa rotata Bieb. & G4.D & - & Ir.-Tur.element & $\mathbf{L C}$ \\
\hline 244 & Dipsacaceae & $\begin{array}{l}\text { Pterocephalus plumosus } \\
\text { (L.) Coulter }\end{array}$ & G4.D & - & Cosmopolitan & LC \\
\hline 245 & Asteraceae & Xanthium spinosum L. & G4.D & - & - & LC \\
\hline 246 & Asteraceae & İnula britannica L. & G4.F & - & $\begin{array}{c}\text { Euro- } \\
\text { Sib.element }\end{array}$ & $\mathbf{L C}$ \\
\hline 247 & Asteraceae & İnula montbretiana DC. & G4.F & - & Ir.-Tur.element & $\mathbf{L C}$ \\
\hline 248 & Asteraceae & $\begin{array}{l}\text { Helichrysum plicatum } \\
\text { DC. subsp. Plicatum }\end{array}$ & G4.D & - & - & $\mathbf{L C}$ \\
\hline 249 & Asteraceae & $\begin{array}{l}\text { Helichrysum arenarium } \\
\text { (L.) Moench subsp. } \\
\text { aucheri (Boiss.) Davis et } \\
\text { Kupicha }\end{array}$ & G4.5 & - & Ir.-Tur.element & LC \\
\hline 250 & Asteraceae & Logfia arvensis (L.) Holub & G4.5 & - & Cosmopolitan & LC \\
\hline 251 & Asteraceae & Senecio mollis Willd. & G4.5 & - & Ir.-Tur.element & $\mathbf{L C}$ \\
\hline 252 & Asteraceae & $\begin{array}{l}\text { Senecio vernalis Waldst. } \\
\text { et Kit. }\end{array}$ & G4.5 & - & Cosmopolitian & LC \\
\hline 253 & Asteraceae & Senecio viscosus L. & G4.F & - & - & LC \\
\hline 254 & Asteraceae & Tussilago farfara L. & C2.6 & - & $\begin{array}{c}\text { Euro- } \\
\text { Sib.element }\end{array}$ & $\mathbf{L C}$ \\
\hline 255 & Asteraceae & $\begin{array}{l}\text { Anthemis tinctoria L. var. } \\
\text { tinctoria }\end{array}$ & G4.F & - & Cosmopolitian & LC \\
\hline 256 & Asteraceae & $\begin{array}{c}\text { Anthemis wiedemanniana } \\
\text { Fisch. et Mey. }\end{array}$ & G4.D & Endemic & - & $\mathbf{V U}$ \\
\hline 257 & Asteraceae & Achillea teretifolia Willd. & G4.F & Endemic & Ir.-Tur.element & VU \\
\hline 258 & Asteraceae & $\begin{array}{c}\text { Achillea setacea Waldst. } \\
\text { et Kit }\end{array}$ & G4.F & - & \begin{tabular}{|c|} 
Euro- \\
Sib.element
\end{tabular} & LC \\
\hline 259 & Asteraceae & $\begin{array}{l}\text { Tanacetum parthenium } \\
\text { (L.) Schultz Bip. }\end{array}$ & G4.F & - & - & LC \\
\hline 260 & Asteraceae & $\begin{array}{l}\text { Tanacetum armenum } \\
\text { (De.) Schultz Bip }\end{array}$ & G4.D & - & - & LC \\
\hline 261 & Asteraceae & Tanacetum vulgare L. & G4.F & - & - & $\mathbf{L C}$ \\
\hline 262 & Asteraceae & $\begin{array}{l}\text { Tripleurospermum } \\
\text { elongatum (Fisch. \& } \\
\text { Mey.) Bornm. } \\
\end{array}$ & G4.F & - & - & LC \\
\hline 263 & Asteraceae & $\begin{array}{c}\text { Tripleurospermum } \\
\text { sevanense (Manden.) } \\
\text { Pobed. }\end{array}$ & G4.F & - & - & LC \\
\hline 264 & Asteraceae & $\begin{array}{l}\text { Arctium minus (Hill) } \\
\text { Bernh. subsp. pubens } \\
\text { (Babington) Arènes }\end{array}$ & C2.6 & - & $\begin{array}{c}\text { Euro- } \\
\text { Sib.element }\end{array}$ & LC \\
\hline
\end{tabular}


Vol.5, No.2, 2019

\begin{tabular}{|c|c|c|c|c|c|c|}
\hline 265 & Asteraceae & $\begin{array}{l}\text { Onopordum turcicum } \\
\text { Danin }\end{array}$ & G4.D & - & Ir.-Tur.element & LC \\
\hline 266 & Asteraceae & $\begin{array}{l}\text { Cirsium vulgare (Savi) } \\
\text { Ten. }\end{array}$ & C2.6 & - & Cosmopolitan & $\mathbf{L C}$ \\
\hline 267 & Asteraceae & $\begin{array}{c}\text { Cirsium arvense (L.) } \\
\text { Scop. subsp. vestitum } \\
\text { (Wimmer et Grab.) Petrak }\end{array}$ & C4.F & - & Cosmopolitan & LC \\
\hline 268 & Asteraceae & $\begin{array}{l}\text { Carduus nutans L. sensu } \\
\text { lato }\end{array}$ & G4.D & - & Cosmopolitan & LC \\
\hline 269 & Asteraceae & $\begin{array}{l}\text { Jurinea pontica Hausskn. } \\
\text { et Freyn }\end{array}$ & G4.5 & Endemic & Ir.-Tur.element & VU \\
\hline 270 & Asteraceae & Centaurea virgata. Lam. & G4.F & - & Ir.-Tur.element & LC \\
\hline 271 & Asteraceae & $\begin{array}{l}\text { Centaurea solstitialis L. } \\
\text { subsp. Solstitialis }\end{array}$ & G4.D & - & Cosmopolitan & LC \\
\hline 272 & Asteraceae & Centaurea iberica Trev. & G4.D & - & - & LC \\
\hline 273 & Asteraceae & $\begin{array}{l}\text { Centaurea urvillei DC. } \\
\text { subsp. stepposa Wagenitz }\end{array}$ & G4.D & - & Ir.-Tur.element & $\mathbf{V U}$ \\
\hline 274 & Asteraceae & $\begin{array}{l}\text { Centaurea pichleri BoIss. } \\
\text { subsp. Pichleri }\end{array}$ & G4.D & - & - & $\mathbf{L C}$ \\
\hline 275 & Asteraceae & Centaurea triumfettii All. & G4.5 & - & Cosmopolitan & $\mathbf{L C}$ \\
\hline 276 & Asteraceae & \begin{tabular}{|l|} 
Crupina (Pers.) \\
crupinastrum (Moris) Vis
\end{tabular} & G4.D & - & - & $\mathbf{L C}$ \\
\hline 277 & Asteraceae & Xeranthemum annuum L. & G4.D & - & Cosmopolitan & $\mathbf{L C}$ \\
\hline 278 & Asteraceae & Echinops galaticus Freyn & G4.D & - & Oxine element & $\mathbf{L C}$ \\
\hline 279 & Asteraceae & Cichorium intybus L. & C2.6 & - & Cosmopolitan & $\mathbf{L C}$ \\
\hline 280 & Asteraceae & $\begin{array}{l}\text { Scorzonera cana } \\
\text { (C.A.Meyer) Hoffm. var. } \\
\text { Cana }\end{array}$ & H3.6 & - & - & LC \\
\hline 281 & Asteraceae & $\begin{array}{c}\text { Scorzonera mollis Bieb. } \\
\text { subsp. szowitzii (DC.) } \\
\text { Chamherlain } \\
\end{array}$ & G4.D & - & Ir.-Tur.element & LC \\
\hline 282 & Asteraceae & $\begin{array}{l}\text { Tragopogon longirostris } \\
\text { Bisch. var. abbreviatus } \\
\text { Boiss. }\end{array}$ & G4.5 & - & - & LC \\
\hline 283 & Asteraceae & $\begin{array}{l}\text { Leontodon hispidus L. } \\
\text { var. Hispidus }\end{array}$ & G4.5 & - & - & LC \\
\hline 284 & Asteraceae & \begin{tabular}{|l|} 
Reichardia glauca \\
Matthews
\end{tabular} & H3.6 & - & Ir.-Tur.element & LC \\
\hline 285 & Asteraceae & \begin{tabular}{|l} 
Hieracium oblongum \\
Jordan
\end{tabular} & G4.D & - & $\begin{array}{c}\text { Euro- } \\
\text { Sib.element }\end{array}$ & LC \\
\hline 286 & Asteraceae & $\begin{array}{l}\text { Hieracium pannosum } \\
\text { Boiss. }\end{array}$ & H3.6 & - & $\begin{array}{c}\text { East } \\
\text { Mediterranian } \\
\end{array}$ & LC \\
\hline 287 & Asteraceae & $\begin{array}{l}\text { Hieracium paphlagonicum } \\
\text { Freyn et Sint. }\end{array}$ & G4.5 & Endemic & - & LC \\
\hline 288 & Asteraceae & $\begin{array}{l}\text { Pilosella hoppeana } \\
\text { (schultes) C.H. et F.W. } \\
\text { Schultz subsp. plisquama } \\
\text { (NP.) Sen et West }\end{array}$ & G4.F & - & Cosmopolitan & LC \\
\hline 289 & Asteraceae & $\begin{array}{l}\text { Pilosella piloselloides } \\
\text { (Vill.) Sojàk subsp. } \\
\text { megalomastix (NP.) Sell } \\
\text { et West }\end{array}$ & G4.5 & - & Cosmopolitan & $\mathbf{L C}$ \\
\hline 290 & Asteraceae & $\begin{array}{l}\text { P1losella x macrotricha } \\
\text { (Boiss.) C.H. et F.W. } \\
\text { Schultz }\end{array}$ & G4.5 & - & Cosmopolitan & LC \\
\hline 291 & Asteraceae & \begin{tabular}{|l|} 
Cephalorrhynchus \\
tuberosus (Stev.) Schchian
\end{tabular} & G4.5 & - & Cosmopolitan & LC \\
\hline 292 & Asteraceae & Lactuca serriola L. & C2.6 & - & $\begin{array}{c}\text { Euro- } \\
\text { Sib.element }\end{array}$ & LC \\
\hline 293 & Asteraceae & $\begin{array}{l}\text { Scariola viminea. (L.) } \\
\text { F.W.Schmidt. }\end{array}$ & G4.5 & - & Cosmopolitan & LC \\
\hline
\end{tabular}


Vol.5, No.2, 2019

\begin{tabular}{|c|c|c|c|c|c|c|}
\hline 294 & Asteraceae & Mycelis muralis (L.) Dum. & G4.5 & - & \begin{tabular}{|l|} 
Euro- \\
Sib.element
\end{tabular} & LC \\
\hline 295 & Asteraceae & $\begin{array}{l}\text { Lapsana communis L. } \\
\text { subsp. alpina (Boiss. et } \\
\text { Bal.) SelI }\end{array}$ & G4.5 & - & $\begin{array}{c}\text { East Black Sea } \\
\text { element }\end{array}$ & $\mathbf{L C}$ \\
\hline 296 & Asteraceae & $\begin{array}{l}\text { Taraxacum serotinum } \\
\text { (Woldst. et Kit.) Poiret }\end{array}$ & C2.6 & - & Cosmopolitan & LC \\
\hline 297 & Asteraceae & $\begin{array}{l}\text { Taraxacum macrolepium } \\
\text { Schischkin }\end{array}$ & C2.6 & - & - & LC \\
\hline 298 & Asteraceae & $\begin{array}{l}\text { Chondrilla juncea L. var. } \\
\text { juncea }\end{array}$ & G4.D & - & - & LC \\
\hline 299 & Asteraceae & $\begin{array}{l}\text { Crepis f'eotida L. subsp. } \\
\text { f'eotida }\end{array}$ & G4.F & - & Cosmopolitan & $\mathbf{L C}$ \\
\hline 300 & Asteraceae & \begin{tabular}{|l|} 
Crepis f'eotida L. \\
Subsepecies \\
rhoeadifolia(Biep.) Celak.
\end{tabular} & G4.F & - & Cosmopolitan & LC \\
\hline 301 & Campanulaceae & $\begin{array}{l}\text { Campanula lyrata Lam. } \\
\text { subsp. Lyrata }\end{array}$ & G4.D & Endemic & - & $\mathbf{V U}$ \\
\hline 302 & Campanulaceae & $\begin{array}{l}\text { Campanula rapunculoides } \\
\text { L. subsp. Rapunculoides }\end{array}$ & G4.5 & - & $\begin{array}{c}\text { Euro- } \\
\text { Sib.element }\end{array}$ & LC \\
\hline 303 & Campanulaceae & $\begin{array}{c}\text { Campanula glomerata L. } \\
\text { subsp. hispida (Witasek) } \\
\text { Hayek } \\
\end{array}$ & G4.5 & - & $\begin{array}{c}\text { Euro- } \\
\text { Sib.element }\end{array}$ & LC \\
\hline 304 & Campanulaceae & $\begin{array}{c}\text { Campanula involucrata } \\
\text { Aucher }\end{array}$ & G4.D & - & Ir.-Tur.element & $\mathbf{L C}$ \\
\hline 305 & Campanulaceae & $\begin{array}{c}\text { Campanula argaea Boiss. } \\
\text { et Bal. }\end{array}$ & H3.6 & Endemic & Ir.-Tur. element & $\mathbf{V U}$ \\
\hline 306 & Campanulaceae & $\begin{array}{c}\text { Campanula cf.reuterena } \\
\text { Boiss. et Bal. }\end{array}$ & G4.D & - & Ir.-Tur.element & LC \\
\hline 307 & Campanulaceae & $\begin{array}{c}\text { Campanula pterocaula } \\
\text { Hausskn. } \\
\end{array}$ & G4.D & Endemic & Oxine element & $\mathbf{V U}$ \\
\hline 308 & Campanulaceae & $\begin{array}{l}\text { Asyneuma rigidum } \\
\text { (Willd.) Grossh. subsp. } \\
\text { Rigidum }\end{array}$ & G4.7 & - & \begin{tabular}{|l|} 
Ir.-Tur.element \\
\end{tabular} & LC \\
\hline 309 & Campanulaceae & $\begin{array}{l}\text { Legousia pentagonia (L.) } \\
\text { Thellung }\end{array}$ & G4.D & - & $\begin{array}{c}\text { East } \\
\text { Mediterranian }\end{array}$ & LC \\
\hline 310 & Ericaceae & $\begin{array}{l}\text { Orthılia secunda (L.) } \\
\text { House }\end{array}$ & G4.D & - & - & LC \\
\hline 311 & Primulaceae & Androsace maxima L. & G4.D & - & Cosmopolitan & $\mathbf{L C}$ \\
\hline 312 & Primulaceae & $\begin{array}{l}\text { Lysimachia verticillaris } \\
\text { Sprenpel }\end{array}$ & C2.6 & - & $\begin{array}{c}\text { East Black Sea } \\
\text { element }\end{array}$ & LC \\
\hline 313 & Primulaceae & $\begin{array}{l}\text { Lysimachia atropurpurea } \\
\text { L. }\end{array}$ & G4.D & - & \begin{tabular}{c|} 
East \\
Mediterranian
\end{tabular} & $\mathbf{L C}$ \\
\hline 314 & Oleaceae & Jasminum fruticans L. & G4.D & - & Mediterranian & $\mathbf{L C}$ \\
\hline 315 & Oleaceae & $\begin{array}{l}\text { Forsythia europaea Degen } \\
\text { et Bald. }\end{array}$ & G3.4 & - & - & LC \\
\hline 316 & Oleaceae & \begin{tabular}{|l} 
Fraxinus angustifolia. \\
Vahl. subsp. angustifolia
\end{tabular} & G3.4 & - & - & LC \\
\hline 317 & Asclepıadaceae & $\begin{array}{l}\text { Vincetoxicum tmoIeum } \\
\text { BoIss. }\end{array}$ & G4.D & - & Ir.-Tur.element & $\mathbf{L C}$ \\
\hline 318 & Convolvulaceae & Convolvulus arvensis L. & C2.6 & - & - & $\mathbf{L C}$ \\
\hline 319 & Cuscutaceae & $\begin{array}{l}\text { Cuscuta epithymum (L.) } \\
\text { L. var. Epithymum }\end{array}$ & G4.9 & - & Cosmopolitan & LC \\
\hline 320 & Boraginaceae & $\begin{array}{l}\text { Heliotropium suaveolens } \\
\text { Bieb. }\end{array}$ & C2.6 & - & \begin{tabular}{c|} 
East \\
Mediterranian \\
\end{tabular} & LC \\
\hline 321 & Boraginaceae & $\begin{array}{l}\text { Rochelia disperma (L.fil.) } \\
\text { C.Koch var. Disperina }\end{array}$ & G4.D & - & Cosmopolitan & $\mathbf{L C}$ \\
\hline 322 & Boraginaceae & $\begin{array}{l}\text { Myosotis alpestris F.W. } \\
\text { Schmidt subsp. Alpestris }\end{array}$ & G4.5 & - & Cosmopolitan & LC \\
\hline 323 & Boraginaceae & $\begin{array}{l}\text { Myosotis lithospermifolia } \\
\text { (Willd.) Hornem. }\end{array}$ & G4.9 & - & - & LC \\
\hline
\end{tabular}


Vol.5, No.2, 2019

\begin{tabular}{|c|c|c|c|c|c|c|}
\hline 324 & Boraginaceae & Myosotis sicula Guss. & G4.D & - & - & $\mathbf{L C}$ \\
\hline 325 & Boraginaceae & $\begin{array}{l}\text { Paracaryum incanum } \\
\text { (Ledeb.) Boiss. }\end{array}$ & G4.D & - & Ir.-Tur.element & $\mathbf{L C}$ \\
\hline 326 & Boraginaceae & $\begin{array}{c}\text { Paracaryum calycinum } \\
\text { Boiss. \& Bal. }\end{array}$ & G4.D & Endemic & $\begin{array}{l}\text { Ir.- } \\
\text { Tur.element }\end{array}$ & LC \\
\hline 327 & Boraginaceae & $\begin{array}{l}\text { Paracaryum ancyritanum } \\
\text { Boiss. }\end{array}$ & G4.D & Endemic & Ir.-Tur.element & LC \\
\hline 328 & Boraginaceae & $\begin{array}{l}\text { Cynoglossum montanum } \\
\text { L. }\end{array}$ & G4.9 & - & $\begin{array}{c}\text { Euro- } \\
\text { Sib.element }\end{array}$ & LC \\
\hline 329 & Boraginaceae & $\begin{array}{l}\text { Buglosso1des arvensis (L.) } \\
\text { Johnston }\end{array}$ & G4.9 & - & Cosmopolitan & LC \\
\hline 330 & Boraginaceae & $\begin{array}{l}\text { Neatostema apulum (L.) } \\
\text { Jonston }\end{array}$ & G4.9 & - & Mediterranian & LC \\
\hline 331 & Boraginaceae & Echium italicum L. & G4.F & - & Mediterranian & $\mathbf{L C}$ \\
\hline 332 & Boraginaceae & $\begin{array}{l}\text { Echium angustifolium } \\
\text { Miller }\end{array}$ & G4.5 & - & $\begin{array}{c}\text { East } \\
\text { Mediterranian }\end{array}$ & LC \\
\hline 333 & Boraginaceae & $\begin{array}{l}\text { Onosma isauricum Boiss. } \\
\text { et Heldr. }\end{array}$ & G4.D & Endemic & Ir.-Tur.element & VU \\
\hline 334 & Boraginaceae & $\begin{array}{l}\text { Cerinthe minor L. subsp. } \\
\text { auriculata. (Ten.) Domac }\end{array}$ & G4.9 & - & Cosmopolitan & LC \\
\hline 335 & Boraginaceae & $\begin{array}{l}\text { Anchusa leptophylla } \\
\text { Roemer et Schultes subsp. } \\
\text { Leptophylla }\end{array}$ & H3.6 & - & - & VU \\
\hline 336 & Solanaceae & Solanum dulcamara L. & H3.4 & - & $\begin{array}{c}\text { Euro- } \\
\text { Sib.element }\end{array}$ & $\mathbf{L C}$ \\
\hline 337 & Solanaceae & Hyoscyamus niger L. & G4.D & - & - & $\mathbf{L C}$ \\
\hline 338 & Scrophulariaceae & $\begin{array}{l}\text { Verbascum flavidum } \\
\text { (Boiss.) Freyn et Bornm. }\end{array}$ & G3.5 & - & $\begin{array}{c}\text { Euro- } \\
\text { Sib.element }\end{array}$ & LC \\
\hline 339 & Scrophulariaceae & $\begin{array}{l}\text { Verbascum armenum } \\
\text { Boiss. et Kotschy var. } \\
\text { tempskyanum (Freyn et } \\
\text { Sint.) Murb. }\end{array}$ & G4.5 & - & - & LC \\
\hline 340 & Scrophulariaceae & $\begin{array}{l}\text { Verbascum armenum } \\
\text { Boiss. et Kotschy var. } \\
\text { occidentale Hub.-Mor. }\end{array}$ & G4.5 & Endemic & Ir.-Tur.element & VU \\
\hline 341 & Scrophulariaceae & $\begin{array}{c}\text { Verbascum insulare Boiss. } \\
\text { et Heldr. }\end{array}$ & G4.5 & Endemic & Ir.-Tur.element & VU \\
\hline 342 & Scrophulariaceae & \begin{tabular}{|c|} 
Verbascum \\
cheiranthifolium BoIss. \\
var. Cheirtmthifolium
\end{tabular} & G4.D & - & - & LC \\
\hline 343 & Scrophulariaceae & $\begin{array}{l}\text { Scrophularia umbrosa } \\
\text { Dum. }\end{array}$ & G4.5 & - & $\begin{array}{c}\text { Euro- } \\
\text { Sib.element }\end{array}$ & LC \\
\hline 344 & Scrophulariaceae & $\begin{array}{l}\text { Scrophularia } \\
\text { xanthoglossa. Boiss. var. } \\
\text { decipiens (Boiss. et } \\
\text { Kotschy) Boiss. } \\
\end{array}$ & G4.5 & - & Ir.-Tur.element & LC \\
\hline 345 & Scrophulariaceae & $\begin{array}{l}\text { Linaria genistifolia (L.) } \\
\text { Miller subsp. confertiflora } \\
\text { (Boiss.) Davis }\end{array}$ & G4.5 & Endemic & Ir.-Tur.element & LC \\
\hline 346 & Scrophulariaceae & $\begin{array}{l}\text { Digitalls ferruginea L. } \\
\text { subsp. Ferruginea }\end{array}$ & G4.5 & - & $\begin{array}{c}\text { Euro- } \\
\text { Sib.elelment }\end{array}$ & LC \\
\hline 347 & Scrophulariaceae & Digitalls lamarckii Ivan & G4.5 & Endemic & Ir.-Tur.element & VU \\
\hline 348 & Scrophulariaceae & $\begin{array}{l}\text { Veronıca pusilla. Kotschy } \\
\text { var. pusilla. }\end{array}$ & G4.D & - & Ir.-Tur.element & LC \\
\hline 349 & Scrophulariaceae & $\begin{array}{l}\text { Veronica anagallis- } \\
\text { aquatica L. }\end{array}$ & C2.6 & - & - & LC \\
\hline 350 & Scrophulariaceae & Veronica chammaedrys L. & G4.D & - & $\begin{array}{c}\text { Euro- } \\
\text { Sib.element }\end{array}$ & $\mathbf{L C}$ \\
\hline 351 & Scrophulariaceae & $\begin{array}{l}\text { Veronica orientalis Miller } \\
\text { subsp. Orientalis }\end{array}$ & G4.D & - & - & LC \\
\hline 352 & Scrophulariaceae & Veronica multifida L. & G4.5 & Endemic & Ir.-Tur.element & VU \\
\hline 353 & Scrophulariaceae & Veronica officinalis L. & G4.5 & - & $\begin{array}{c}\text { Euro- } \\
\text { Sib.element }\end{array}$ & $\mathbf{L C}$ \\
\hline
\end{tabular}


Vol.5, No.2, 2019

\begin{tabular}{|c|c|c|c|c|c|c|}
\hline 354 & Scrophulariaceae & Euphrasia pectinata Ten. & G4.5 & - & $\begin{array}{c}\text { Euro- } \\
\text { Sib.element }\end{array}$ & VU \\
\hline 355 & Scrophulariaceae & $\begin{array}{l}\text { Pedicularis comosa L. var. } \\
\text { sibthorpii (Boiss.) Boiss. }\end{array}$ & G4.D & - & - & LC \\
\hline 356 & Orobanchaceae & Orobanche purpurea Jacq. & C2.6 & - & Cosmopolitan & LC \\
\hline 357 & Globulariaceae & $\begin{array}{l}\text { Globularia trichosantha } \\
\text { Fisch. et Mey. }\end{array}$ & G4.D & - & Ir.-Tur.element & LC \\
\hline 358 & Lamiaceae & $\begin{array}{l}\text { Teucrium orientale L. var. } \\
\text { Orientale }\end{array}$ & G4.5 & - & Ir.-Tur.element & LC \\
\hline 359 & Lamiaceae & $\begin{array}{l}\text { Teucrium chamaedrys L. } \\
\text { subsp. Chamaedrys }\end{array}$ & G4.5 & - & $\begin{array}{c}\text { Euro- } \\
\text { Sib.element }\end{array}$ & LC \\
\hline 360 & Lamiaceae & Teucrium polium L. & H3.6 & - & - & LC \\
\hline 361 & Lamiaceae & $\begin{array}{l}\text { Scutellariai albida L. } \\
\text { subsp. albida }\end{array}$ & G4.5 & - & $\begin{array}{c}\text { East } \\
\text { Mediterranian }\end{array}$ & LC \\
\hline 362 & Lamiaceae & $\begin{array}{l}\text { Scutellariai orientalis L. } \\
\text { subsp. pinnatifida } \\
\text { Edmondson }\end{array}$ & G4.5 & - & - & LC \\
\hline 363 & Lamiaceae & Phlomis armeniaca Willd. & G4.5 & Endemic & Ir.-Tur.element & VU \\
\hline 364 & Lamiaceae & $\begin{array}{l}\text { Lamium purpureum L. } \\
\text { var. Purpureum }\end{array}$ & G4.D & - & \begin{tabular}{c|} 
Euro- \\
Sib.element
\end{tabular} & LC \\
\hline 365 & Lamiaceae & Lamıum album L. & G4.5 & - & $\begin{array}{c}\text { Euro- } \\
\text { Sib.element }\end{array}$ & LC \\
\hline 366 & Lamiaceae & $\begin{array}{l}\text { Ballota nigra L. subsp. } \\
\text { anatolica P.H. Davis }\end{array}$ & H3.6 & Endemic & Ir.-Tur.element & $\overline{L C}$ \\
\hline 367 & Lamiaceae & Marrubium vulgare L. & H3.6 & - & - & $\mathbf{L C}$ \\
\hline 368 & Lamiaceae & $\begin{array}{l}\text { Sideritis } \\
\text { germanicopolitana Bornm. } \\
\text { subsp. germanicopolitana }\end{array}$ & G4.5 & Endemic & - & LC \\
\hline 369 & Lamiaceae & Stachys byzantina C.Koch & G4.5 & - & $\begin{array}{c}\text { Euro- } \\
\text { Sib.element }\end{array}$ & $\mathbf{L C}$ \\
\hline 370 & Lamiaceae & $\begin{array}{l}\text { Stachys iberica Bieb. } \\
\text { subsp. stenostachya } \\
\text { (Boiss.) Rech. }\end{array}$ & G4.7 & - & Ir.-Tur.element & LC \\
\hline 371 & Lamiaceae & $\begin{array}{l}\text { Stachys annua (L.) L. } \\
\text { subsp. annua var. } \\
\text { lycaonica Bhattacharjee }\end{array}$ & G4.5 & - & Ir.-Tur.element & VU \\
\hline 372 & Lamiaceae & $\begin{array}{l}\text { Nepeta nuda L. subsp. } \\
\text { Nuda }\end{array}$ & G4.D & - & Cosmopolitan & LC \\
\hline 373 & Lamiaceae & Prunella vulgaris L. & C2.6 & - & $\begin{array}{c}\text { Euro- } \\
\text { Sib.element }\end{array}$ & $\mathbf{L C}$ \\
\hline 374 & Lamiaceae & $\begin{array}{l}\text { Clinopodium vulgare L. } \\
\text { subsp. Vulgare }\end{array}$ & G4.7 & - & $\begin{array}{c}\text { Euro- } \\
\text { Sib.element }\end{array}$ & LC \\
\hline 375 & Lamiaceae & Acinos rotundifolius Pers. & G4.D & - & Cosmopolitan & LC \\
\hline 376 & Lamiaceae & $\begin{array}{l}\text { Thymus sibthorpii } \\
\text { Bentham }\end{array}$ & G3.C & - & $\begin{array}{c}\text { Euro- } \\
\text { Sib.element }\end{array}$ & LC \\
\hline 377 & Lamiaceae & $\begin{array}{l}\text { Thymus sipyleus Boiss. } \\
\text { subsp. rosulans (Barbas) } \\
\text { Jalas }\end{array}$ & H3.6 & - & - & VU \\
\hline 378 & Lamiaceae & $\begin{array}{l}\text { Mentha longifolia (L.) } \\
\text { Hudson subsp. typhoides } \\
\text { (Briq) Harley var. } \\
\text { Typhoides } \\
\end{array}$ & G4.8 & - & - & LC \\
\hline 379 & Lamiaceae & Ziziphora capitata L. & G4.D & - & Ir.-Tur.element & $\mathbf{L C}$ \\
\hline 380 & Lamiaceae & Salvia tomentosa Miller & G4.D & - & Mediterranian & $\mathbf{L C}$ \\
\hline 381 & Lamiaceae & Salvia sclarea L. & G4.9 & - & - & LC \\
\hline 382 & Lamiaceae & $\begin{array}{l}\text { Salvia candidissima Vahl. } \\
\text { subsp. occidentalis Hedge }\end{array}$ & H3.6 & - & Ir.-Tur.element & LC \\
\hline 383 & Lamiaceae & Salvia virgata Jacq. & G4.D & - & Ir.-Tur.element & $\mathbf{L C}$ \\
\hline 384 & Lamiaceae & $\begin{array}{l}\text { Salvia verticillata. L. } \\
\text { subsp. Verticillata }\end{array}$ & G4.9 & - & $\begin{array}{c}\text { Euro- } \\
\text { Sib.element }\end{array}$ & $\mathrm{LC}$ \\
\hline 385 & Plumbaginaceae & Plumbago europae L. & $\mathrm{C2.6}$ & - & $\begin{array}{c}\text { Euro- } \\
\text { Sib.element }\end{array}$ & LC \\
\hline
\end{tabular}


Vol.5, No.2, 2019

\begin{tabular}{|c|c|c|c|c|c|c|}
\hline 386 & Plumbaginaceae & $\begin{array}{l}\text { Acantholımon glumaceum } \\
\text { (Jaub. et Spach.) Boiss. }\end{array}$ & G4.7 & - & Ir.-Tur.element & LC \\
\hline 387 & Plumbaginaceae & $\begin{array}{l}\text { Acantholimon ulicinum } \\
\text { (Willd. ex Schultes) Boiss. } \\
\text { subsp. lycaonicum (Boiss. } \\
\text { et Heldr.) Bokhari et } \\
\text { Edmondson }\end{array}$ & G4.7 & - & Ir.-Tur.element & LC \\
\hline 388 & Plantaginaceae & $\begin{array}{l}\text { Plantago major L. subsp. } \\
\text { Majör }\end{array}$ & C2.6 & - & - & LC \\
\hline 389 & Plantaginaceae & Plantago holosteum Scop. & G4.9 & - & Mediterranian & $\mathbf{L C}$ \\
\hline 390 & Plantaginaceae & Plantago lanceolata. L. & G4.F & - & Cosmopolitan & $\mathbf{L C}$ \\
\hline 391 & Santalaceae & Thesium billardieri Boiss. & G4.7 & - & Ir.-Tur.element & $\mathbf{L C}$ \\
\hline 392 & Loranthaceae & $\begin{array}{l}\text { Viscum album L. subsp. } \\
\text { austriacum (Wiesb.) } \\
\text { Wollman }\end{array}$ & G4.D & - & - & LC \\
\hline 393 & Euphorbiaceae & Euphorbia stricta L. & G4.8 & - & $\begin{array}{c}\text { Euro- } \\
\text { Sib.element }\end{array}$ & LC \\
\hline 394 & Euphorbiaceae & $\begin{array}{l}\text { Euphorbıa szovitsii Fisch. } \\
\& \text { Mey. var szovitsii }\end{array}$ & G4.8 & - & Ir.-Tur.element & $\mathbf{L C}$ \\
\hline 395 & Euphorbiaceae & $\begin{array}{l}\text { Euphorbia falcata L. } \\
\text { subsp. falcata var. Falcata }\end{array}$ & G4.8 & - & Ir.-Tur.element & $\mathbf{L C}$ \\
\hline 396 & Euphorbiaceae & Euphorbıa myrsinites L. & G4.D & - & - & $\mathbf{L C}$ \\
\hline 397 & Urticaceae & Urtica dioica $\mathrm{L}$ & C2.6 & - & $\begin{array}{c}\text { Euro- } \\
\text { Sib.element }\end{array}$ & LC \\
\hline 398 & Urticaceae & Parietaria judaica L. & G4.7 & - & Cosmopolitan & LC \\
\hline 399 & Moraceae & Morus alba L & G3.4 & - & - & $\mathbf{L C}$ \\
\hline 400 & Ulmaceae & $\begin{array}{l}\text { Ulmus minor Miller } \\
\text { subsp. minor }\end{array}$ & G4.9 & - & - & $\mathbf{L C}$ \\
\hline 401 & Platanaceae & Platanus orientalis L. & G4.9 & - & - & $\mathbf{L C}$ \\
\hline 402 & Fagaceae & $\begin{array}{l}\text { Quercus macranthera } \\
\text { Fisch. et Mey. subsp. } \\
\text { syspirensis (C.Koch) } \\
\text { Menitsky }\end{array}$ & G4.7 & - & - & LC \\
\hline 403 & Fagaceae & \begin{tabular}{|l|} 
Quercus petraea \\
(Mattuschka) Liebl. subsp. \\
iberica (Steven ex Bieb.) \\
Krassiln.
\end{tabular} & G4.7 & - & - & $\mathbf{L C}$ \\
\hline 404 & Fagaceae & Quercus pubescens Willd. & G4.7 & - & - & LC \\
\hline 405 & Corylaceae & Carpınus betulus L. & G4.9 & - & $\begin{array}{c}\text { Euro- } \\
\text { Sib.element }\end{array}$ & LC \\
\hline 406 & Corylaceae & $\begin{array}{l}\text { Corylus avellana L. var. } \\
\text { avellana }\end{array}$ & G4.9 & - & $\begin{array}{c}\text { Euro- } \\
\text { Sib.element }\end{array}$ & $\mathbf{L C}$ \\
\hline 407 & Salicaceae & Salix alba L. $q$ & G4.D & - & $\begin{array}{c}\text { Euro- } \\
\text { Sib.element }\end{array}$ & $\mathbf{L C}$ \\
\hline 408 & Salicaceae & Salix babylonica L. $\hat{O}^{\lambda}$ & G3.4 & - & - & LC \\
\hline 409 & Salicaceae & Salix caprea. L. & G4.D & - & $\begin{array}{c}\text { Euro- } \\
\text { Sib.element }\end{array}$ & $\mathbf{L C}$ \\
\hline 410 & Salicaceae & Populus tremula. L. & G4.D & - & $\begin{array}{c}\text { Euro- } \\
\text { Sib.element }\end{array}$ & LC \\
\hline 411 & Salicaceae & $\begin{array}{l}\text { Populus nigra L. subsp. } \\
\text { Nigra }\end{array}$ & G4.D & - & - & LC \\
\hline 412 & Rubiaceae & $\begin{array}{l}\text { Crucianella bithynica. } \\
\text { Boiss. }\end{array}$ & G4.D & - & $\begin{array}{c}\text { East } \\
\text { Mediterranian }\end{array}$ & LC \\
\hline 413 & Rubiaceae & $\begin{array}{l}\text { Asperula involucrata. } \\
\text { Wahlenb. }\end{array}$ & G4.D & - & Oxine element & LC \\
\hline 414 & Rubiaceae & $\begin{array}{l}\text { Galium verum L. subsp. } \\
\text { Verum }\end{array}$ & G4.7 & - & $\begin{array}{c}\text { Euro- } \\
\text { Sib.element }\end{array}$ & LC \\
\hline 415 & Rubiaceae & $\begin{array}{l}\text { Galium verum L. Subsp. } \\
\text { glabrescens Ehrend. }\end{array}$ & G4.C & - & Ir.-Tur.element & LC \\
\hline 416 & Rubiaceae & Galium lovcense Urumov & G4.7 & - & - & $\mathbf{L C}$ \\
\hline 417 & Rubiaceae & $\begin{array}{l}\text { Galium fissurense Ehrend. } \\
\text { et Schönb. }\end{array}$ & G4.7 & Endemic & Oxine element & $\mathbf{V U}$ \\
\hline
\end{tabular}


Vol.5, No.2, 2019

\begin{tabular}{|c|c|c|c|c|c|c|}
\hline 418 & Rubiaceae & $\begin{array}{l}\text { Galium incanum subsp. } \\
\text { elatius (Boiss.) Ehrend }\end{array}$ & G4.D & - & Ir.-Tur. element & LC \\
\hline 419 & Rubiaceae & $\begin{array}{l}\text { Galıum penduliflorum } \\
\text { Boiss. }\end{array}$ & G4.D & Endemic & $\begin{array}{c}\text { East } \\
\text { mediterranian }\end{array}$ & LC \\
\hline 420 & Rubiaceae & $\begin{array}{l}\text { Cruciata taurica (Pallas ex } \\
\text { Willd.) Ehrend. }\end{array}$ & H3.6 & - & Ir.-Tur. element & LC \\
\hline 421 & Liliaceae & Eremurus spectabilis Bieb. & G4.7 & - & Ir.-Tur. element & $\mathbf{L C}$ \\
\hline 422 & Liliaceae & $\begin{array}{l}\text { Allium huber-morathii } \\
\text { Kollmann, N.Özhatay \& } \\
\text { Koyuncu }\end{array}$ & G4.7 & Endemic & Ir.-Tur. element & LC \\
\hline 423 & Liliaceae & $\begin{array}{l}\text { Allium scorodoprasum L. } \\
\text { subsp. rotundum (Le) } \\
\text { Stearn }\end{array}$ & G4.7 & - & Mediterranian & LC \\
\hline 424 & Liliaceae & Allium vineale L. & G4.7 & - & - & LC \\
\hline 425 & Liliaceae & Allium lycaonicum Siehe & G4.7 & - & - & $\mathbf{L C}$ \\
\hline 426 & Liliaceae & Scilla bifolia L. & G4.D & - & Mediterranian & $\mathbf{L C}$ \\
\hline 427 & Liliaceae & \begin{tabular}{|l|} 
Ornithogalum \\
oligophyllum E.D. Clarke
\end{tabular} & G4.7 & - & - & LC \\
\hline 428 & Liliaceae & $\begin{array}{l}\text { Ornithogalum umbellatum } \\
\text { L. }\end{array}$ & G4.5 & - & - & LC \\
\hline 429 & Liliaceae & \begin{tabular}{|l|} 
Ornithogalum \\
armeniacum Baker
\end{tabular} & G4.D & - & $\begin{array}{c}\text { East } \\
\text { mediterranian }\end{array}$ & LC \\
\hline 430 & Liliaceae & $\begin{array}{l}\text { Muscari comosum (L.) } \\
\text { Miller }\end{array}$ & G4.D & - & Mediterranian & LC \\
\hline 431 & Liliaceae & $\begin{array}{l}\text { Muscari aucheri (Boiss.) } \\
\text { Baker }\end{array}$ & G4.D & Endemic & - & VU \\
\hline 432 & Liliaceae & $\begin{array}{l}\text { Muscari armeniacum } \\
\text { Leichtlin }\end{array}$ & G4.D & - & - & $\mathbf{L C}$ \\
\hline 433 & Liliaceae & Tulipa sintenisii Baker & G4.5 & Endemic & Ir.-Tur. element & $\mathbf{L C}$ \\
\hline 434 & Liliaceae & Gagea luteoides Stapf. & G4.5 & - & - & $\mathbf{L C}$ \\
\hline 435 & Liliaceae & $\begin{array}{l}\text { Gagea peduncularis (J.\& } \\
\text { C.Persl) Pascher }\end{array}$ & G4.D & - & Mediterranian & LC \\
\hline 436 & Liliaceae & $\begin{array}{l}\text { Colchicum szovitsii Fisch. } \\
\text { et Mey }\end{array}$ & G4.D & - & $\begin{array}{l}\text { Ir.-Tur. } \\
\text { element }\end{array}$ & LC \\
\hline 437 & Amaryllıdaceae & $\begin{array}{l}\text { Galanthus elwesli Hooker } \\
\text { subsp. tuebitaki N.Zeybek }\end{array}$ & G4.7 & - & $\begin{array}{c}\text { East } \\
\text { mediterranian } \\
\text { montain }\end{array}$ & $\mathbf{L C}$ \\
\hline 438 & Iridaceae & $\begin{array}{l}\text { Crocus ancyrensis } \\
\text { (Herbert) Maw }\end{array}$ & G4.D & Endemic & $\begin{array}{l}\text { Ir.-Tur. } \\
\text { element }\end{array}$ & $\mathbf{V U}$ \\
\hline 439 & Iridaceae & $\begin{array}{l}\text { Crocus biflorus Miller } \\
\text { subsp. pulchricolor } \\
\text { (Herbert) Mathew }\end{array}$ & G4.D & Endemic & $\begin{array}{c}\text { Euro-Sib. } \\
\text { element }\end{array}$ & LC \\
\hline 440 & Orchidaceae & $\begin{array}{l}\text { Orchis mascula (L.) L. } \\
\text { subsp. pinetorum (Boiss. } \\
\text { et Kotschy). G.Camus }\end{array}$ & G4.5 & - & $\begin{array}{c}\text { East } \\
\text { mediterranian }\end{array}$ & LC \\
\hline 441 & Juncaceae & Juncus inflexus L. & G1.2 & - & Cosmopolitan & LC \\
\hline 442 & Juncaceae & $\begin{array}{l}\text { Juncus gerardi Loisel. } \\
\text { subsp. Gerardi }\end{array}$ & G1.2 & - & - & $\mathbf{L C}$ \\
\hline 443 & Juncaceae & Juncus articulatus L. & C2.6 & - & $\begin{array}{c}\text { Euro- } \\
\text { Sib.element }\end{array}$ & LC \\
\hline 444 & Cyperaceae & $\begin{array}{l}\text { Eleocharıs palustris (L.) } \\
\text { Roemer et Sehultes }\end{array}$ & G1.2 & - & - & LC \\
\hline 445 & Cyperaceae & Carex divisia Hudson & G1.2 & - & $\begin{array}{c}\text { Euro-Sib. } \\
\text { element }\end{array}$ & LC \\
\hline 446 & Cyperaceae & Carex spicata Hudson & G1.2 & - & $\begin{array}{c}\text { Euro-Sib. } \\
\text { Element }\end{array}$ & $\mathbf{L C}$ \\
\hline 447 & Cyperaceae & $\begin{array}{l}\text { Carex melanostachya } \\
\text { Bieb. }\end{array}$ & G1.2 & - & Cosmopolitan & LC \\
\hline 448 & Poaceae & $\begin{array}{l}\text { Brachypodium sylvaticum } \\
\text { (Hudson) P.Beauv. }\end{array}$ & G1.2 & - & $\begin{array}{c}\text { Euro-Sib. } \\
\text { element }\end{array}$ & LC \\
\hline 449 & Poaceae & Elymus caninus (L.) L. & H3.6 & - & $\begin{array}{c}\text { Euro-Sib. } \\
\text { element }\end{array}$ & LC \\
\hline
\end{tabular}


Vol.5, No.2, 2019

\begin{tabular}{|c|c|c|c|c|c|c|}
\hline 450 & Poaceae & \begin{tabular}{|l} 
Elymus hispidus (Opiz) \\
Melderis subsp. Hispidus
\end{tabular} & G4.7 & - & Cosmopolitan & LC \\
\hline 451 & Poaceae & $\begin{array}{l}\text { Aegilops umbellulata. } \\
\text { Zhukovsky subsp. } \\
\text { umbellulata. }\end{array}$ & G4.D & - & $\begin{array}{l}\text { Ir.-Tur. } \\
\text { element }\end{array}$ & LC \\
\hline 452 & Poaceae & Hordeum bulbosum L. & G4.D & - & Cosmopolitan & LC \\
\hline 453 & Poaceae & Bromus danthoniae Trin. & G4.7 & - & - & $\mathbf{L C}$ \\
\hline 454 & Poaceae & Bromus tectorum L. & G4.D & - & - & LC \\
\hline 455 & Poaceae & $\begin{array}{l}\text { Bromus ramosus } \\
\text { Hudson }\end{array}$ & G1.2 & - & - & LC \\
\hline 456 & Poaceae & $\begin{array}{l}\text { Arrhenatherum elatius } \\
\text { (L.) Beauv. subsp. } \\
\text { elatius. }\end{array}$ & G4.5 & - & $\begin{array}{c}\text { Euro-Sib. } \\
\text { element }\end{array}$ & LC \\
\hline 457 & Poaceae & $\begin{array}{l}\text { Koeleria cristata (L.) } \\
\text { Pers. }\end{array}$ & H3.6 & - & $\begin{array}{c}\text { Cosmopolita } \\
\text { n }\end{array}$ & LC \\
\hline 458 & Poaceae & $\begin{array}{l}\text { Calamagrostis } \\
\text { pseudophragmites } \\
\text { (Haner fil.) Koeler }\end{array}$ & G4.D & - & $\begin{array}{c}\text { Euro-Sib. } \\
\text { element }\end{array}$ & LC \\
\hline 459 & Poaceae & $\begin{array}{l}\text { Apera spica-venti (L.) } \\
\text { P.Beauv. }\end{array}$ & H3.6 & - & $\begin{array}{l}\text { Euro-Sib. } \\
\text { element }\end{array}$ & LC \\
\hline 460 & Poaceae & Agrostis stolonifera L. & G1.2 & - & $\begin{array}{c}\text { Euro-Sib. } \\
\text { element }\end{array}$ & LC \\
\hline 461 & Poaceae & $\begin{array}{l}\text { Allopecurus aequalis } \\
\text { Sobol. }\end{array}$ & G1.2 & - & $\begin{array}{c}\text { Euro-Sib. } \\
\text { element }\end{array}$ & LC \\
\hline 462 & Poaceae & $\begin{array}{c}\text { Allopecurus } \\
\text { arundineceus Poiret }\end{array}$ & G4.5 & - & $\begin{array}{c}\text { Euro-Sib. } \\
\text { element }\end{array}$ & $\mathbf{L C}$ \\
\hline 463 & Poaceae & $\begin{array}{l}\text { Allopecurus textilis } \\
\text { Boiss. subsp. Textilis }\end{array}$ & G4.5 & - & $\begin{array}{l}\text { Ir.-Tur. } \\
\text { element }\end{array}$ & LC \\
\hline 464 & Poaceae & Phleum bertolonii DC. & G4.5 & - & $\begin{array}{c}\text { Cosmopolita } \\
\text { n }\end{array}$ & LC \\
\hline 465 & Poaceae & $\begin{array}{l}\text { Festuca valesiaca } \\
\text { Schleicher }\end{array}$ & G4.7 & - & $\begin{array}{c}\text { Cosmopolita } \\
\mathbf{n}\end{array}$ & LC \\
\hline 466 & Poaceae & $\begin{array}{l}\text { Festuca callieri } \\
\text { (Hackel) F.Markgraf } \\
\text { subsp. zederbaueri } \\
\text { Markgr.-Dannenb. } \\
\end{array}$ & G4.D & Endemic & $\begin{array}{l}\text { Ir.-Tur. } \\
\text { element }\end{array}$ & LC \\
\hline 467 & Poaceae & Poa pratensis L. & G4.D & - & $\begin{array}{c}\text { Cosmopolita } \\
\mathbf{n}\end{array}$ & $\mathbf{L C}$ \\
\hline 468 & Poaceae & Poa angustifolia. L. & G4.D & - & $\begin{array}{c}\text { Cosmopolita } \\
\text { n }\end{array}$ & LC \\
\hline 469 & Poaceae & Poa bulbosa L. & G4.D & - & $\begin{array}{c}\text { Cosmopolita } \\
\text { n }\end{array}$ & LC \\
\hline 470 & Poaceae & $\begin{array}{l}\text { Dactylis glomerata L. } \\
\text { subsp. hispanica (Roth) } \\
\text { Nyman }\end{array}$ & G4.7 & - & - & LC \\
\hline 471 & Poaceae & Briza humilis Bieb. & G4.7 & - & - & LC \\
\hline 472 & Poaceae & $\begin{array}{l}\text { Melica ciliata L. subsp. } \\
\text { Ciliata }\end{array}$ & G4.9 & - & - & $\mathrm{LC}$ \\
\hline 473 & Poaceae & $\begin{array}{l}\text { Glyceria plicata. (Fries) } \\
\text { Fries }\end{array}$ & G4.5 & - & - & LC \\
\hline 474 & Poaceae & Stipa holosericea Trin. & H3.6 & - & $\begin{array}{l}\text { Ir.-Tur. } \\
\text { element }\end{array}$ & LC \\
\hline 475 & Poaceae & $\begin{array}{l}\text { Stipa pulcherrimia } \\
\text { C.Koch. subsp. epilosa } \\
\text { (Martinovsky) Tzvelev }\end{array}$ & H3.6 & - & - & $\mathbf{L C}$ \\
\hline 476 & Poaceae & $\begin{array}{l}\text { Piptatherum holciforme } \\
\text { (Bieb.) Roemer et } \\
\text { Schultes subsp. } \\
\text { holciforme var. } \\
\text { Holciforme }\end{array}$ & G4.D & - & - & LC \\
\hline 477 & Poaceae & $\begin{array}{l}\text { Setaria verticillata (L.) } \\
\text { P.Beauv. var. ambigua. } \\
\text { (Guss.) ParI }\end{array}$ & G4.D & - & - & LC \\
\hline
\end{tabular}




\begin{tabular}{|l|l|l|c|c|c|c|}
\hline 478 & Poaceae & $\begin{array}{l}\text { Pennisetum orientale } \\
\text { L.C.M. Richard }\end{array}$ & G4.D & - & $\begin{array}{l}\text { Ir.-Tur. } \\
\text { element }\end{array}$ & LC \\
\hline
\end{tabular}

\section{CONCLUSION}

The results of this study are based on the examination of 1064 plant samples collected in a two year period and field observations and literature search on the subject. As a result of naming of the plant samples, a total of 481 taxons were determined including 276 genera, 474 species, 4 subspecies, 3 varieties belonging to 74 families. 49 species (10.6\%) out of 474 species were endemic. 2 plant species collected belonged to PTERIDOPHYTA Division and 472 species belonged to SPERMATOPHYTA Division.

The distribution of the species in the study area by phyto-geographic regions is shown in Table 9, the phyto-geographic region spectrum is shown in Figure 3, the first ten families consisting of the most number of species are shown in Table 110, the family spectrum is shown in Figure 4, the comparison of the first ten families consisting of the most number of species with other studies is shown in Table 11, the comparison of the first ten genera consisting of the most number of species is shown in Table 12, the distribution of the study area and other studies by phyto-geographic regions is shown in Table 113, and our samples showing morphological differences according to the flora are shown in Table 14.

The plant geographical region with the most number of species in our area was European-Siberian region (82 species), and the other regions were in turn Iranian-Turanian region (76 species), and the Mediterranean region (31 species). 292 species showed wide distribution or belonged to no specific region (Table 9, Figure 3).

The family that was rich in terms of species number in our study area was Asteraceae. Fabaceae, Poaceae, Lamiaceae and Brassicaceae families were the first 5 families rich in terms of species number. The genus having the most number of species in the area was Vicia. The second genus was Trifalium, the third genus was Silene, the fourth genera was Sedum, and the fifth genera was Campanula. Among these, Vicia is widespread in particularly under the non-evergreen forests and the glades. Trifolium and Campanula are widespread in such type of forests. Sedum was represented by rich number of species because of the abundant number of rocks and stony areas located in the area.

Table9. Distribution of the species by phyto-geopraphical regions

\begin{tabular}{lcrr} 
Phyto-geographical Region & & Number of Species & Ratio \% \\
\cline { 3 - 4 } European - Siberian & 71 & 14,8 \\
Black Sea & 11 & 2,3 \\
Iranian-Turanian & 76 & 15,8 \\
Mediterranean & 31 & 6,5 \\
Multiple regions and unknown & 292 & 60,6
\end{tabular}

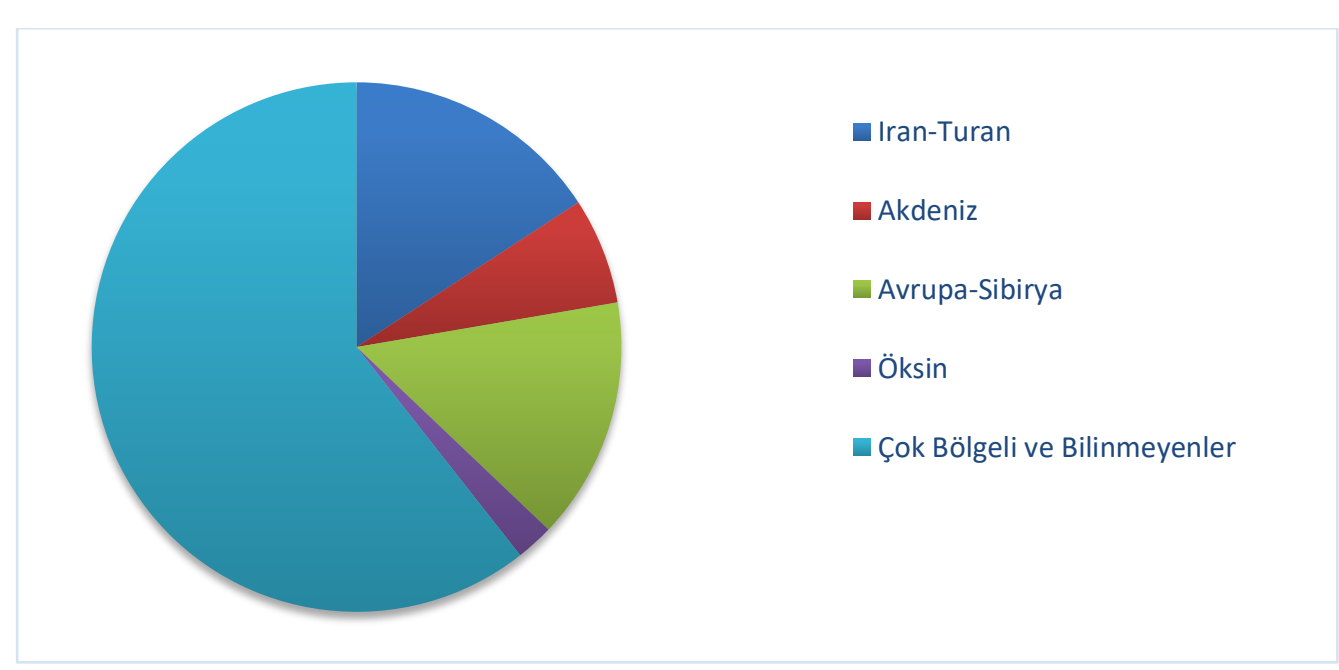

Iranian-Turanian / Mediterranean / European-Siberian / Siberian-Oxin / Multiple regions and unknown Figure3. Phtogeographical region spectrum of the species 
Table10. The first 10 families consisting of the most number of species

\section{Family Name}

$\underline{\%}$

Asteraceae

Fabaceae

Poaceae

Lamiaceae

Brassicaceae

Rosaceae

Caryophyllaceae

Apiaceae

Boraginaceae

Scrophulariaceae

Total

Remaining 63 Families

\section{$\underline{\text { Number of Species }}$}

55

50

31

27

24

24

23

26

16

16

289

185

\section{$\underline{\text { Ratio of the Total Number of Species }}$}

11,6
10,6
6,6
5,7
5,0
5,0
4,9
4,9
3,4
3,4

61,1
38,9

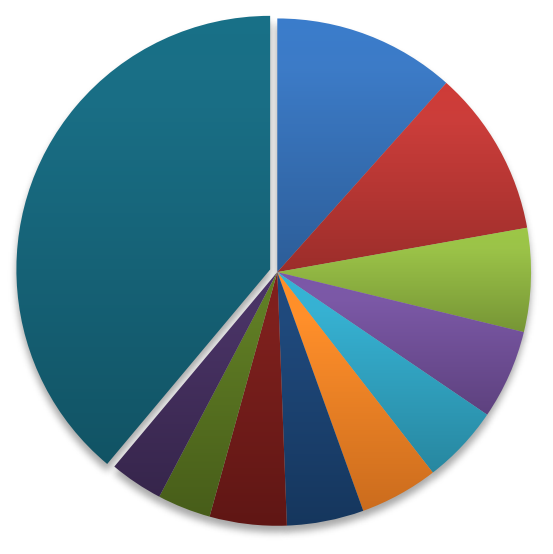

- Asteraceae

- Fabaceae

Poaceae

- Lamiaceae

- Brassicaceae

- Rosaceae

- Caryophyllaceae

- Apiaceae

- Boraginaceae

- Scrophulariaceae

- Diğer Familyalar

Figure4. Family spectrum

The plant samples collected by other researchers, not by us, in the study area and its vicinity are listed below. Though the exact locations were not specified in the majority of the labels of these plant samples, it can be concluded that the samples were collected in areas in the vicinity of our area or within our area.

- Ophioglossum vulgatum L.

A4 Ankara: Kızılcahamam, Kühne 1312

- Asplenium septentrionale (L.) Hoffm.

A4 Ankara: Kızılcahamam 1840 m, Karamanoğlu 5700

- Sagina procumbens L.

A4 Ankara: Kızılcahamam, Kühne 1312

- Astragalus squalidus Boiss. \& Nöe in Boiss.

A4 Ankara: Kızılcahamam, 1340 m, Hub.-Mor. 12896

- Trifoliiim retusum L.

A4 Ankara: d. Kızılcahamam, M.Zohary 51341

- Trifolium patens Schreb.

A4 Ankara: Kızılcahamam, 1350 m, Alpay (ANKO2625)

- Trifolium physodes Stev.

A4 Ankara: Kizılcahamam, Orshan 51331

- Trifolium ochroleucum Huds 
Vol.5, No.2, 2019

A4 Ankara: K1zılcahamam forest M. Zohary 51342

- Crataegus orientalis Pallas var. orientalis

A4 Ankara: Kızılcahamam, Uluocak ve Tammuz (ISTO 1090)

- Bidens tripartita L..

A4 Ankara: Kızılcahamam, Müller 198

- Taraxaciim androssovii Schischkin

A4 Ankara: N-of Kızılcahamam, 1300 m, Sorger 71.3.12

- Crepis pulchra L. subsp. pulchra

A4 Ankara: Kızılcahamam, Birand et M.Zohary 3374

- Anchusa barrelieri (All.) Vitman var. barellieri

A4 Ankara: Kızılcahamam, 1100-1400 m, Khan et al 693

- Serophularia. lucida L.

A4 Ankara: Kızılcahamam to Soğuksu, Leblebici ve Ersoy

(EGE 12621)

- Linaria corifolia Desf.

A4 Ankara: Kizılcahamam, T.Baytop (ISTE 13392)

- Orobanche mutelli F.Schultz

A4 Ankara: Kizılcahamam, 1960, Fitz

- Stachys macrantha (C.Koch) Stearn

A4 Ankara: nr Kizilcahamam, Bilger 5214

- Nepeta italica L.

A4 Ankara: Kızılcahamam, Birand et Zohary 3379

- Najas minor All.

A4 Ankara: Kızılcahamam, 100 m, A.Baytop (ISTE 40867 p.p)

- Potamogeton panormitanus Biv.

A4 Ankara: Kizılcahamam, 1000 m, A.Baytop (ISTE 40871)

- Lemna gibba L.

A4 Ankara: Kizılcahamam, 1000 ID, A.Baytop (ISTE 40869 p.p)

- Lemna. minor L.

A4 Ankara: K1zılcahamam, A.Baytop (ISTE 27121)

- Colchicum bornmuelleri Freyn

A4 Ankara: Kızılcahamam, 15.IX.1940, Kasapligil

- Crocus danfordiae Maw

A4 Ankara: Kızılcahamam, 1500 ID, Mathew et Tomlinson 4053

- Epipactis eondensata Boiss.

A4 Ankara: Soğuksu Milli Parkı, 1300 ID, A. et. C.Nieschalk 1124

\section{DISCUSSION}

As a result of the comparison of our results obtained in the study area and the results of the previous studies conducted in the places in the vicinity of the area, the situation regarding the families having the most number of species is as follows:

The richest family in our area was Asteraceae family. This family was in the second rank generally in all other studies. According to the results of the study conducted at Ayaş Mountains and Beypazar1-Nallihan region, these two regions were in the third rank. These two regions are different than our region in terms of formation and step formation is dominant in those regions in general. Due to the characteristics of these two regions, Poaceae family, which is common in step fields, was ranked second in these regions and this family was in the third rank in our area. Despite there was a difference between our area and the other areas in terms of the families listed in the first 10 ranks, the names were similar. Though the names of the families listed in the first 5 ranks were generally identical, there were some differences in the listing. However, in the studies conducted in the vicinity of Gerede-Aktaş and KızılcahamamKargasekmez, Scrophulariaceae family ranked the $3^{\text {rd }}$ and $5^{\text {th }}$. This family ranked the $10^{\text {th }}$ in our area. This family was in the $3^{\text {rd }}$ rank in the vicinity of Gerede-Aktas because Veronica genus belonging to this family was the richest genus in this area and because this area had unique features. Considering the genera of the vicinity of Gerede-Aktas (Table 121), it is clear that the rich genera of this area had some characteristics different than the other areas. The genus having the most number of species in our area was Vicia with 11 species. The glades and tensile area situated in our area are the cause for the richness of this genus that is widespread in such type of forests. This genus was the $5^{\text {th }}$ richest genus at Ayas Mountain, having a typical forest destruction field and covered with dwarf oaks. The second richest genus 
in our area was Trifolium and it ranked the second in the vicinity of Gerede-Aktas, Beypazar1-Nallihan and again the second in the vicinity of Kizilcahamam-Kargasekmez. The first 2 areas, particularly Gerede-Aktas environs were similar to our area in terms of formation and the dominant tree species. Quercus was dominant in the vicinity of Kargasekmez. It is natural that Quercus was widespread in both our area and in the dense and thin forests made up by other forest trees, and Trifolium genus, which is the most common genus in such type of habitats, was represented by abundant number of species. Another remarkable issue among the rich genera was that Astragalus, which ranked the first in the floras of Beypazar1-Nallihan and Beynam, ranked the $7^{\text {th }}$ in our area, and it ranked the $6^{\text {th }}$ in Gerede-Aktas, which was a similar area. Another remarkable issue was that Salvia ranked first in some other studies and it was not listed in the first 10 in our area. This was due to the commonness of this genus in step areas in general. Centaurea genus, which is again common in step areas, ranked the $9^{\text {th }}$ in our area due to the same reason. Another interesting situation in terms of rich genera occurs for Sedum. This genus ranked the $4^{\text {th }}$ in both our area and Gerede-Aktas, which is similar to our area and in the vicinity of our area. The richness of this genus in our area can be explained with the quite abundance of rocks, which is the growing environment of this genus, in our area. Elatine alsinastrum, the most interesting genus and species collected in our area, grows in Göllü locality, covered with water until midsummer and drying up in July. Though this genus seems to be new in Anatolia according to the record of Flora of Turkey, it was reported in A9 grid by Donner. This species was recorded in Denizli as well. However, this species was recorded in Thrace in the book titled Med-Checq List. Thus, determination of this species in our area is the second record in Anatolia. However, many researchers might have missed the fact that the plant is very short and it grows in aquatic habitats. Therefore, we hope that this product will widespread in Turkey through carefully conducted future studies.

Table11. Comparison of the first ten families consisting of the most number of species with other studies $(\%)$

\begin{tabular}{|c|c|c|c|c|c|c|c|c|}
\hline \multicolumn{3}{|c|}{$\begin{array}{l}\text { CONDUCTED } \\
\text { STUDIES }\end{array}$} & $\begin{array}{l}\text { S.M.P } \\
\text { (A4) }\end{array}$ & $\begin{array}{l}\text { Gerede-Aktaş } \\
\text { (A4) }\end{array}$ & $\begin{array}{l}\text { Kizılcahamam- } \\
\text { Kargasekmez } \\
\text { (A4) }\end{array}$ & $\begin{array}{l}\text { Ayaş } \\
\text { (A4) }\end{array}$ & $\begin{array}{l}\text { Beypazar1- } \\
\text { Nallihan } \\
\text { (A4) }\end{array}$ & $\begin{array}{l}\text { Beynam } \\
\text { (A4) }\end{array}$ \\
\hline \multicolumn{3}{|c|}{$\begin{array}{l}\text { CONDUCTED } \\
\text { STUDIES }\end{array}$} & 1 & 2 & 3 & 4 & 5 & 6 \\
\hline \multicolumn{3}{|c|}{$\begin{array}{l}\text { FAMİLY NAME } \\
\text { TOT. SPECIES S. } \\
\text { RATE }(\%) \\
\end{array}$} & $\begin{array}{c}\text { Asteraceae } \\
11,6\end{array}$ & $\begin{array}{c}\text { Fabaceae } \\
11,1\end{array}$ & $\begin{array}{c}\text { Fabaceae } \\
16,4\end{array}$ & $\begin{array}{c}\text { Fabaceae } \\
12,0\end{array}$ & $\begin{array}{c}\text { Fabaceae } \\
12,0\end{array}$ & $\begin{array}{c}\text { Fabaceae } \\
12,8\end{array}$ \\
\hline י" & י" & י & $\begin{array}{c}\text { Fabaceae } \\
10,6\end{array}$ & $\begin{array}{c}\text { Asteraceae } \\
8,5\end{array}$ & $\begin{array}{c}\text { Asteraceae } \\
9,9\end{array}$ & $\begin{array}{c}\text { Poaceae } \\
8,8\end{array}$ & $\begin{array}{c}\text { Poaceae } \\
9,0\end{array}$ & $\begin{array}{c}\text { Asteraceae } \\
10,2\end{array}$ \\
\hline י" & " & י" & $\begin{array}{c}\text { Poaceae } \\
6,6\end{array}$ & $\begin{array}{c}\text { Scrophulariaceae } \\
8,2\end{array}$ & $\begin{array}{c}\text { Poaceae } \\
7,9\end{array}$ & $\begin{array}{c}\text { Asteraceae } \\
8,3\end{array}$ & $\begin{array}{c}\text { Asteraceae } \\
7,6 \\
\end{array}$ & $\begin{array}{c}\text { Lamiaceae } \\
8,3\end{array}$ \\
\hline " & יי & י" & $\begin{array}{c}\text { Lamiaceae } \\
5,7\end{array}$ & $\begin{array}{c}\text { Poaceae } \\
5,3\end{array}$ & $\begin{array}{c}\text { Lamiaceae } \\
7,5\end{array}$ & $\begin{array}{c}\text { Lamiaceae } \\
8,1\end{array}$ & $\begin{array}{c}\text { Brassicaceae } \\
6,8\end{array}$ & $\begin{array}{c}\text { Poaceae } \\
6,2\end{array}$ \\
\hline י" & יי & $"$ & $\begin{array}{c}\text { Brassicaceae } \\
5,0\end{array}$ & $\begin{array}{c}\text { Lamiaceae } \\
5,0\end{array}$ & $\begin{array}{c}\text { Scrophulariaceae } \\
5,0\end{array}$ & $\begin{array}{c}\text { Brassicaceae } \\
5,5\end{array}$ & $\begin{array}{c}\text { Lamiaceae } \\
6,0\end{array}$ & $\begin{array}{c}\text { Rosaceae } \\
5,4\end{array}$ \\
\hline "י & יי & יי & $\begin{array}{c}\text { Rosaceae } \\
5,0\end{array}$ & $\begin{array}{c}\text { Rosaceae } \\
5,0\end{array}$ & $\begin{array}{c}\text { Brassicaceae } \\
4,8\end{array}$ & $\begin{array}{c}\text { Caryophyllaceae } \\
5,5\end{array}$ & $\begin{array}{c}\text { Caryophyllaceae } \\
4,7\end{array}$ & $\begin{array}{c}\text { Caryophyllaceae } \\
4,5\end{array}$ \\
\hline "י & י" & י" & $\begin{array}{c}\text { Caryophyllaceae } \\
4,9\end{array}$ & $\begin{array}{c}\text { Caryophyllaceae } \\
5,0\end{array}$ & $\begin{array}{c}\text { Apiaceae } \\
4,2\end{array}$ & $\begin{array}{c}\text { Scrophulariaceae } \\
4,3\end{array}$ & $\begin{array}{c}\text { Rosaceae } \\
4,2\end{array}$ & $\begin{array}{c}\text { Brassicaceae } \\
4,2\end{array}$ \\
\hline יי & יי & י" & $\begin{array}{c}\text { Apiaceae } \\
4,9\end{array}$ & $\begin{array}{c}\text { Barassicaceae } \\
4,7\end{array}$ & $\begin{array}{c}\text { Caryophyllaceae } \\
3,7\end{array}$ & $\begin{array}{c}\text { Boraginaceae } \\
3,8\end{array}$ & $\begin{array}{c}\text { Scrophulariaceae } \\
3,7\end{array}$ & $\begin{array}{c}\text { Boraginaceae } \\
3,8\end{array}$ \\
\hline " & "י & יי & $\begin{array}{c}\text { Boraginaceae } \\
3,4\end{array}$ & $\begin{array}{c}\text { Boraginaceae } \\
4,4\end{array}$ & $\begin{array}{c}\text { Boraginaceae } \\
3,7\end{array}$ & $\begin{array}{c}\text { Apiaceae } \\
3,5\end{array}$ & $\begin{array}{c}\text { Boraginaceae } \\
2,5\end{array}$ & $\begin{array}{c}\text { Apiaceae } \\
3,5\end{array}$ \\
\hline י" & יי & יי & $\begin{array}{c}\text { Scrophulariaceae } \\
3,4\end{array}$ & $\begin{array}{c}\text { Apiaceae } \\
3,1\end{array}$ & $\begin{array}{c}\text { Rosaceae } \\
3,7\end{array}$ & $\begin{array}{c}\text { Rosaceae } \\
2,7\end{array}$ & $\begin{array}{c}\text { Apiaceae } \\
2\end{array}$ & $\begin{array}{c}\text { Scrophulariaceae } \\
3,5\end{array}$ \\
\hline
\end{tabular}


International Journal of Scientific and Technological Research ISSN 2422-8702 (Online), DOI: 10.7176/JSTR/5-2-43

Vol.5, No.2, 2019

ISt?

Table 12. Comparison of the first 10 genera containing the most number of species with the other studies

\begin{tabular}{|c|c|c|c|c|c|c|c|c|}
\hline \multicolumn{3}{|c|}{$\begin{array}{l}\text { CONDUCTED } \\
\text { STUDIES }\end{array}$} & $\begin{array}{l}\text { S.M.P } \\
\text { (A4) }\end{array}$ & Gerede-Aktaş & $\begin{array}{l}\text { Kizılcahamam- } \\
\text { Kargasekmez }\end{array}$ & Ayaş & $\begin{array}{l}\text { Beypazar1- } \\
\text { Nallihan }\end{array}$ & Beynam \\
\hline \multicolumn{3}{|c|}{$\begin{array}{l}\text { TOTAL NUMBER } \\
\text { OF SPECIES }\end{array}$} & 474 & 315 & 451 & 387 & 616 & 419 \\
\hline \multicolumn{3}{|c|}{$\begin{array}{c}\text { GENUS NAME } \\
\text { AND SPECIES } \\
\text { CONTAINED } \\
\text { NUMBER OF } \\
\text { SPECIES }\end{array}$} & $\begin{array}{l}\text { Vicia } \\
11\end{array}$ & $\begin{array}{l}\text { Veronica } \\
12\end{array}$ & $\begin{array}{l}\text { Trifolium } \\
13\end{array}$ & $\begin{array}{c}\text { Astragalus } \\
\quad 21\end{array}$ & $\begin{array}{c}\text { Astragalus } \\
18\end{array}$ & $\begin{array}{c}\text { Astragalus } \\
23\end{array}$ \\
\hline י & י & $"$ & $\begin{array}{l}\text { Trifolium } \\
8\end{array}$ & $\begin{array}{l}\text { Trifolium } \\
9\end{array}$ & $\begin{array}{c}\text { Astragalus } \\
11\end{array}$ & $\begin{array}{l}\text { Silene } \\
9\end{array}$ & $\begin{array}{l}\text { Trifolium } \\
14\end{array}$ & $\begin{array}{l}\text { Salvia } \\
9\end{array}$ \\
\hline י & י & $"$ & $\begin{array}{l}\text { Silene } \\
8\end{array}$ & $\begin{array}{c}\text { Lathyrus } \\
7\end{array}$ & $\begin{array}{c}\text { Ranunculus } \\
8 \\
\end{array}$ & $\begin{array}{l}\text { Salvia } \\
8 \\
\end{array}$ & $\begin{array}{c}\text { Galium } \\
11 \\
\end{array}$ & $\begin{array}{l}\text { Silene } \\
9\end{array}$ \\
\hline " & י & $"$ & $\begin{array}{c}\text { Sedum } \\
7 \\
\end{array}$ & $\begin{array}{c}\text { Sedum } \\
7 \\
\end{array}$ & $\begin{array}{c}\text { Silene } \\
8 \\
\end{array}$ & $\begin{array}{c}\text { Alyssum } \\
7 \\
\end{array}$ & $\begin{array}{c}\text { Galium } \\
11 \\
\end{array}$ & $\begin{array}{l}\text { Silene } \\
9 \\
\end{array}$ \\
\hline יי & י & "י & $\begin{array}{c}\text { Campanala } \\
6 \\
\end{array}$ & $\begin{array}{c}\text { Ranunculus } \\
6 \\
\end{array}$ & $\begin{array}{c}\text { Veronica } \\
8 \\
\end{array}$ & $\begin{array}{c}\text { Vicia } \\
7 \\
\end{array}$ & $\begin{array}{c}\text { Alyssum } \\
10 \\
\end{array}$ & $\begin{array}{c}\text { Centaurea } \\
6 \\
\end{array}$ \\
\hline י & י" & $"$ & $\begin{array}{c}\text { Alyssum } \\
6\end{array}$ & $\begin{array}{l}\text { Astragalus } \\
6\end{array}$ & $\begin{array}{c}\text { Hypericum } \\
7 \\
\end{array}$ & $\begin{array}{c}\text { Anthemis } \\
7\end{array}$ & $\begin{array}{l}\text { Silene } \\
9 \\
\end{array}$ & $\begin{array}{c}\text { Hieracium } \\
5 \\
\end{array}$ \\
\hline י & י & $"$ & $\begin{array}{c}\text { Astragalus } \\
6 \\
\end{array}$ & $\begin{array}{c}\text { Campanula } \\
6 \\
\end{array}$ & $\begin{array}{c}\text { Verbascum } \\
7 \\
\end{array}$ & $\begin{array}{c}\text { Euphorbia } \\
7 \\
\end{array}$ & $\begin{array}{l}\text { Veronica } \\
9 \\
\end{array}$ & $\begin{array}{c}\text { Ranunculus } \\
5 \\
\end{array}$ \\
\hline י & י & י & $\begin{array}{l}\text { Lathyrus } \\
6 \\
\end{array}$ & $\begin{array}{c}\text { Myosotis } \\
6 \\
\end{array}$ & $\begin{array}{c}\text { Galium } \\
7\end{array}$ & $\begin{array}{l}\text { Trifolium } \\
6 \\
\end{array}$ & $\begin{array}{c}\text { Bromus } \\
9\end{array}$ & $\begin{array}{c}\text { Galium } \\
5\end{array}$ \\
\hline י & י" & י & $\begin{array}{c}\text { Centaurea } \\
6 \\
\end{array}$ & $\begin{array}{l}\text { Hypericum } \\
5 \\
\end{array}$ & $\begin{array}{l}\text { Alyssum } \\
5 \\
\end{array}$ & $\begin{array}{c}\text { Carex } \\
6 \\
\end{array}$ & $\begin{array}{c}\text { Epilobium } \\
7 \\
\end{array}$ & $\begin{array}{c}\text { Hypericum } \\
4 \\
\end{array}$ \\
\hline י & י & " & $\begin{array}{c}\text { Veronica } \\
6 \\
\end{array}$ & $\begin{array}{l}\text { Galium } \\
5 \\
\end{array}$ & $\begin{array}{l}\text { Sedum } \\
5 \\
\end{array}$ & $\begin{array}{l}\text { Galium } \\
5 \\
\end{array}$ & $\begin{array}{c}\text { Campanula } \\
6 \\
\end{array}$ & $\begin{array}{c}\text { Trifolium } \\
4 \\
\end{array}$ \\
\hline
\end{tabular}

Table 13. Distribution of the study area and other studies by phyto-geographical regions

\begin{tabular}{|c|c|c|c|c|c|}
\hline $\begin{array}{l}\text { STUDY } \\
\text { AREA }\end{array}$ & $\begin{array}{l}\text { S.M.P } \\
(1990)\end{array}$ & $\begin{array}{l}\text { Gerede-Aktaş } \\
\quad(19799\end{array}$ & $\begin{array}{c}\text { Kizilcahamam- } \\
\text { Kargasekmez } \\
\text { (1979) }\end{array}$ & $\begin{array}{l}\text { Ayaş } \\
(1979)\end{array}$ & $\begin{array}{c}\text { Beypazar1-Nallihan } \\
\text { (1974) }\end{array}$ \\
\hline $\begin{array}{c}\text { Plant } \\
\text { Geographical } \\
\text { Region }\end{array}$ & $\begin{array}{l}\text { European-Sib } \\
17,1 \% \\
\text { Ir-Tur } 15,8 \% \\
\text { Mediterranen } \\
6,5 \%\end{array}$ & $\begin{array}{l}\text { European-Sib } \\
7,8 \% \\
\text { Ir-Tur. 7,6\% } \\
\text { Mediterranean } \\
7,6 \%\end{array}$ & $\begin{array}{l}\text { Ir-Tur. \%11,9 } \\
\text { Mediterranean } \\
\% 11,7 \\
\text { Euro-Sib \%6,1 }\end{array}$ & $\begin{array}{l}\text { Ir-Tur \%23,3 } \\
\text { Mediterranean } \\
\% 14,7 \\
\text { Euro-Sib \%3,5 }\end{array}$ & $\begin{array}{l}\text { Mediterranean \%20,3 } \\
\text { Ir.-Tur. } \% 17 \\
\text { Euro-Sib. } \% 3,7\end{array}$ \\
\hline
\end{tabular}


Table14. Species differing in terms of their morphological characteristics

\begin{tabular}{|c|c|c|}
\hline Species Name & Characteristic of the Flora & Characteristic of the Sample \\
\hline$\overline{\text { Silene fabaria }}$ & $\begin{array}{l}\text { Base leaves are obovat } \\
\text { Calyx is not bulged. }\end{array}$ & $\begin{array}{l}\text { Taban yapraklar spatulat } \\
\text { Calyx is bulged. }\end{array}$ \\
\hline Ferulago galbanifera & $\begin{array}{l}\text { Bractea is } 3-5 \mathrm{~mm} \\
\text { Rays are } 4-8\end{array}$ & $\begin{array}{c}\text { Bractea is } 6,8 \mathrm{~mm} \\
\text { Rays are } 10-12\end{array}$ \\
\hline Neatostema apulum & Stem is $8-22 \mathrm{~cm}$ & Stem is taller than $22 \mathrm{~cm}$, it is $45 \mathrm{~cm}$ \\
\hline Campanula cf. Reuterena & $\begin{array}{l}\text { Branched stem } \\
\text { Corolla is maximum } 35 \mathrm{~mm}\end{array}$ & $\begin{array}{l}\text { Branched stem } \\
\text { Corolla is } 40 \mathrm{~mm}\end{array}$ \\
\hline Plantago holosteum & Pedicle is maximum $14 \mathrm{~cm}$ & Pedicle can be $18 \mathrm{~cm}$ \\
\hline Muscari aucheri & Pedicel is maximum $5 \mathrm{~mm}$ & Pedicel can be $8 \mathrm{~mm}$ \\
\hline
\end{tabular}

Comparison of the plant samples collected in our area and in its vicinity in terms of their geographical locations and components does not provide concrete results since the studies on Beypazar1-Nallihan were published in 1974 and the other studies were published in 1979. The first 4 volumes of the Flora of Turkey were published in 1974 and the first 6 volumes were published in 1979. Therefore, the ratios provided in these studies may not be very precise.

In our area, the European-Siberian region contained the most number of species and the second richest region was the Iranian-Turanian region and the last region was the Mediterranean. This order was identical to Gerede-Aktas as mentioned since its conditions were similar to that of our area. Mediterranean-origin plants dominated in Beypazar1-Nallihan, Iranian-Turanian-origin plants dominated in Ayas, and Iranian-Turanian-origin plants dominated in Kızılcahamam-Kargasekmez. Considering the ecological conditions of the areas where those studies were conducted, these results are normal but, the ratios are not consistent with each other. For example, the ratios of Iranian-Turanian and Mediterranean components in Kizilcahamam-Kargasekmez were almost identical and this may not be quite accurate considering the location of the area. There was a similar situation in Gerede-Aktas as well. Despite the number of European-Siberian components was higher in this area in comparison to the other areas, this difference was $0.2 \%$.

We think that these results are not very accurate. It is certain that these ratios will change with the evaluation of the plant samples collected in this area today when the flora publication is completed. With this study, we hope that the floristic composition of one of our National Parks has been put forward and that we have contributed to the flora of Turkey.

\section{REFERENCES}

[1] Ankara Regional Directorate of Forestry, Kızılcahamam Department of Forestry, "National Park Special Amenajman Plan of Soğuksu National Park Regional Department" Ankara, (1989).

[2] Akman, Y., Aydoğdu, M., "A Phytosociological study in the vicinilies of Çamlıdere Çamkoru and Peçenek" Comm fac. Sci.Univ. Ser C: v.4.pp. 9-19, Ankara (1986).

[3] Akman, Y., Ketenoğlu, O., Contribution to the Flora of Ișık Mauntain and KızılcahamamKargasekmez Region, Comm. Fae. Sci. Univ. Ank. Ser C 2 (Bot) 23, Supple:3, (1979). 
[4] Akman, Y., Ketenoğlu, O., Flora of The Gerede-Aktas Forest, Comm.Fac. Sci. Univ. Ank. Ser. C 2 (Bot) 23, (1979).

[5] Akman, Y., Ketenoğlu, O., Contribution d létude de la flore des montagnes d'Ayas, Comm. Fac. Sci. Univ. Ank. Ser C2 (Bot) 23: Supple, (1979).

[6] Akman, Y., Flora of the Beynam Forest, Comm.Fac.Sci.Univ.Ank. Ser C. 16, (1972).

[7] Akman, Y., " Contribution a l'étutde de la flore de la region de Beypazarı Karaşar et de Nallıhan" Comm. Fac.Sci.Univ Ser.C: Sci. NatureL., 18.C: 8-9, Ankara,1974).

[8] Başbakanlık Devlet Meteoroloji İsleri Genel Müdürlüğü, Ortalama, Extrem Sıcaklık ve YağıȘ Değerleri Bülteni, Ankara (1984).

[9] Brunner, H., Tanker, N., Mesleki Latince, Ank.ün. Eczacılık Fak., 47, Ankara, (1978).

[10]Davis, P.H., Flora of Turkey and The East Aegean Islands Vol:1I-10, Edinburgh, Univ.Press, (1965-88).

[11]Donner, J., Verbreitungskarten zu P.H. Davis, Flora of Turkey, 9, Linzer Biol. Beitr, 19/1, 316, Linz, (1987).

[12] Donner, J., "Verbreitungskarten zu P.H. Davis, Flora of Turkey, 9" Linzer Biol. Beitr. 19/1, 3 16, Linz, (1987).

[13] Erik, S., "New Plant Species in A4 Grid Determined in Ankara, Karagöl (Çubuk) Environs and Central Anatolia Region”, Bitki, Volume:3, Issue:3, 270-276 Ankara (1976).

[14] Erik, S., Demirkuş, N.,"New Distribution Areas of Some Plants in Flora of Turkey" Nature, Botany, 12 (3), 224-233, Ankara (1988).

[15]Eyüboğlu,Ö., (A4) new floristic records for Anatolia, Herbaceous Systematic Botany Journal, Volume 1, Issue 2, year 1994, Ankara

[16] Eyüboğlu, Ö., Our Endemic Plants Determined in Kızılcahamam Soğuksu National Park, Forestry Engineering, Issue 2, year 1997, Ankara.

[17] Evan, G., Townsend, C.C., Flora of Iraq. Vol.9,Baghdad, (19G8)

[18] Greuter, W., Burdet, H.H., Long, G., Hed-checkHst, Vol 1,3,4, Conservatoire et Jardin Botaniques de la Ville de Geneve (1984-1989).

[19] Heywood, V.H., Tutin, G.T. (ed.), Flora Europaea, Vol: I-V, Cambridge Univ. Press. (19641981).

[20] Heywood, V.H. (Ed.), Flawering Plants Of The World, Univ. Press., London, (1978).

[21] Hutchinson, J., The Genere Of Flowering Plants (Angiospermae), Dicotyledones, Vol. 1, Oxford, (1964).

[22] Ketenoğlu, O.,Aydoğdu, M.,"New Floristic Records for Anatolia $\left(A_{3}, A_{4}, A_{5}\right)$ " Nature. Botany, 11(1), 87-93, Ankara, (1987).

[23] Nezahat Gökyiğit, List of Endangered Plant Species of Botanical Garden and ANG Foundation. A4 Grid according to Grid System

[24] Radford, A.E., Dickison, W.C., et al. Vascular Plant Systematics, Taxonomy New York, (1974). 
Vol.5, No.2, 2019

[25] Seçmen.,Ö., Leblebici., E., "Aguatic flora of Western Anatolia", Willdenowia, 14, (1984)

[26] Stearn, T.W., Botanical Latin, Edinburg London (1966).

[27] Yıldırıml, S., "New Floristic Records for Various Grids for Turkey" Nature, TU. Journal of Botany, Volume 11, Issue 1, pp.197, (1987).

[28] Yıldırımlı, Ş., Güner, A., "New Floristic Records for Various Grids for Turkey" Natural, Botany, 13 (2), 321-328, Ankara, (1989) 